$\underline{\text { Preprint typeset in JHEP style - HYPER VERSION }}$

DAMTP-2009-12

CERN-PH-TH/2009-014

\title{
Metastable SUSY Breaking, de Sitter Moduli Stabilisation and Kähler Moduli Inflation
}

\author{
Sven Krippendorf ${ }^{1}$, Fernando Quevedo ${ }^{1,2}$ \\ ${ }^{1}$ DAMTP, Centre for Mathematical Sciences, \\ Wilberforce Road, Cambridge, CB3 OWA, United Kingdom \\ ${ }^{2}$ CERN PH-TH, CH 1211, Geneva 23, Switzerland.
}

\begin{abstract}
We study the influence of anomalous $U(1)$ symmetries and their associated D-terms on the vacuum structure of global field theories once they are coupled to $\mathcal{N}=1$ supergravity and in the context of string compactifications with moduli stabilisation. In particular, we focus on a IIB string motivated construction of the ISS scenario and examine the influence of one additional $U(1)$ symmetry on the vacuum structure. We point out that in the simplest one-Kähler modulus compactification, the original ISS vacuum gets generically destabilised by a runaway behaviour of the potential in the modulus direction. In more general compactifications with several Kähler moduli, we find a novel realisation of the LARGE volume scenario with D-term uplifting to de Sitter space and both D-term and F-term supersymmetry breaking. The structure of soft supersymmetry breaking terms is determined in the preferred scenario where the standard model cycle is not stabilised non-perturbatively and found to be flavour universal. Our scenario also provides a purely supersymmetric realisation of Kähler moduli (blow-up and fibre) inflation, with similar observational properties as the original proposals but without the need to include an extra (non-SUSY) uplifting term.
\end{abstract}

Keywords: dS vacua in string theory, Strings and branes phenomenology, Supersymmetry Breaking, Inflation. 


\section{Contents}

1. Introduction 2

2. The ISS model and its embedding into String Theory 3

3. The One-modulus Case 5

4. The Two-moduli Case 7

4.1 Strategy 8

4.2 Matter field stabilisation 9

4.3 Stabilising the Kähler moduli 12

5. D7 Soft-Terms 15

6. Kähler moduli inflation 19

7. 3-Parameter K3 fibration and Fibre Inflation 21

7.1 Fibre Inflation 23

8. Constraints for metastable dS vacua in supergravity setups 25

8.1 Constraints on Anomalous $U(1)$ Gauge Symmetries as Uplifting Potential 26

9. Conclusions 26

A. On stabilising the F-term potential with a constraint 27

B. Next to leading order corrections to $\Phi \quad 28$

G. Minimizing quark masses 29

D. Estimating scales 29

E. An approximative analytic stabilisation of the large Kähler modulus 31

F. Calculating F-terms 32 


\section{Introduction}

In this article we address the following issues related to supersymmetry breaking, moduli stabilisation, de Sitter vacua and moduli inflation in string compactifications:

1. Despite the recent great success on moduli stabilisation in string theory, the value of the vacuum energy after moduli stabilisation naturally corresponds to anti de Sitter space. This is understandable from the effective field theory point of view due to the fact that the scalar potential of $\mathcal{N}=1$ supergravity is not positive definite and therefore the local minima tend to be at negative values of the vacuum energy. The original uplifting mechanism to de Sitter space proposed in reference [1] by introducing anti D3 branes requires the explicit breaking of supersymmetry in the effective field theory. Further uplifting mechanisms have been proposed [2,3]. Despite partial success, there is not at the moment a compelling mechanism for de Sitter uplifting. The consideration of D-terms as proposed in [2] has to be implemented in concrete setups since, even though D-terms add a positive definite contribution to the effective potential, it is known that if the F-terms vanish then D-terms also vanish [4]. Concrete examples have been provided in field theoretical [5] and string inspired models [6,7].

2. In the past two years there has been a large amount of work on the natural appearance of metastable supersymmetry breaking vacua in global supersymmetry and in particular, the breakdown of global supersymmetry can be achieved significantly simpler. This is the ISS scenario [8]. Some realisations of this mechanism in string models have also been obtained [9]. Nevertheless, there is an implicit assumption in this mechanism, i.e. that the gauge coupling of the corresponding gauge theory is constant and therefore the dynamical non-perturbative scale $\Lambda \sim e^{-a / g^{2}}$ appears as a constant in the effective action. In string theory, however, the gauge coupling is field dependent $1 / g^{2} \sim \operatorname{Re} T$ with $T$ the complex scalar component of a chiral superfield corresponding to a closed string modulus. Therefore the exponential dependence of $\Lambda$ would tend to give a runaway behaviour to the scalar potential as a function of $\operatorname{Re} T$.

3. In the past few years several string theory mechanisms for cosmological inflation have been proposed. The inflaton field corresponds to either an open string mode as in brane-antibrane [10], D3/D7 [11], monodromy [12] or Wilson line [13] inflation or a closed string modulus as in Racetrack [14,15], Kähler moduli [16,17], monodromy [18] or fibre [19] inflation. In most of these scenarios, the realisation of inflation depends crucially on the uplifting mechanism for de Sitter moduli stabilisation. Therefore it tends to go beyond the $\mathcal{N}=1$ supersymmetric effective action if the uplifting mechanism is the presence of anti D3 branes as in KKLT. It would be desirable to find a string inflation mechanism derived from a fully $\mathcal{N}=1$ supersymmetric action. Recently in [20], general constraints on this possibility have been found, substantially restricting the class of supersymmetric models that can give rise to inflation. It is a challenge to find a concrete stringy realisation of inflation that satisfies those constraints. 
We address these three issues by considering a class of models that can be realised in terms of D-brane orientifold constructions in fluxed Calabi-Yau manifolds. In particular we consider a system of magnetised D7 branes with chiral matter fields. The existence of anomalous $U(1)$ 's induces Fayet-Iliopoulos D-terms. Fluxes of RR form give rise to a tunable constant term in the superpotential and also the presence of matter fields provides a non-perturbative superpotential. Depending on the number of D-branes this can be realised in the electric or magnetic phase of SQCD. The electric phase was considered in [6]. Here we will concentrate on the magnetic phase. This makes contact with the ISS models [8] and then naturally addresses the issue of the runaway behaviour of the scalar potential. We find that in the simplest case of one single Kähler modulus, instead of a metastable vacuum, the potential runs away in the direction of the modulus corresponding to the size of the 4-cycle wrapped by the magnetised D7 branes, ruining the interesting properties of the ISS mechanism. However, once we have several Kähler moduli, the situation changes drastically. We find minima of the scalar potential at finite values of the fields with supersymmetry broken as in the ISS mechanism. Furthermore, depending on the values of the free parameters, the minima correspond to either anti de Sitter or de Sitter spaces. In the latter case, it provides a natural uplifting mechanism for moduli stabilisation, addressing also the first issue mentioned above.

Even though the presence of matter fields and D-terms changes the structure of the scalar potential very much, we find remarkably that, once the matter fields are integrated out, the scalar potential for moduli fields ends up to be very similar, but not identical, to the one found in $[21,22]$ and provides a new realisation of the LARGE volume scenario of moduli stabilisation.

Due to the similarity with the original LARGE volume scenario, we derive the structure of soft supersymmetry breaking terms and compare with previous results, we then revisit the Kähler moduli and fibre inflation scenario in our setup and find that they are also naturally realised, therefore providing a pure $\mathcal{N}=1$ realisation of these inflationary scenarios.

\section{The ISS model and its embedding into String Theory}

We want to discuss the vacuum structure of the magnetic dual within the Seiberg duality setup of SQCD which arises in the range $N_{C}<N_{F}<3 / 2 N_{C}$. As commonly known in this phase of SQCD there exists a dual description of the low-energy field theory in terms of an infrared-free modified version of SQCD, the so-called free magnetic dual description. It is characterised by the meson fields $\Phi$, quarks $q$ and anti-quarks $p$ which transform under the following symmetry groups:



where the only gauge symmetry is the $S U\left(N_{F}-N_{C}\right)$ symmetry. The Kähler potential is 
taken to be canonical and looks like

$$
K=|q|^{2}+|p|^{2}+|\Phi|^{2}
$$

In order to obtain dynamical SUSY breaking one introduces a mass term. Hence the most general invariant superpotential we can write down is

$$
W=\Lambda \frac{q \Phi p}{\mu}+\Lambda m \Phi
$$

where $m$ denotes the mass parameter of our theory, $\Lambda$ the dynamical scale of our theory, and $\mu$ is another parameter that is determined by the duality relations. In the above equations and in the whole article we omit indices where possible. Please note that the scale $\Lambda$ has to be introduced due to dimensional requirements.

The metastable vacuum solution is given by $\Phi=0$ and $p=q=i \sqrt{\mu m}$. A derivation can be found in [23]. The mass term explicitly breaks the $U(1)_{A}$ global symmetry.

There have been several realisations of the ISS scenario within string theory in terms of different brane configurations [9]. Here we are more interested in capturing the main ingredients that a string theory realisation will add to this scenario, namely the fact that the parameters $m$ and $\Lambda$ have to be dynamical variables. In particular $\Lambda$ in string theory having a non-perturbative origin is of the form $\Lambda \sim e^{-a T}$ with $T$ a closed string modulus. One simple explicit realisation is the magnetic version of the case considered in [6]. The basic setup is an orientifold model consisting of a stack with $N_{C}+1$ branes, from which one has a non-vanishing magnetic flux leading to $U\left(N_{c}\right) \times U(1)$ gauge theory. The open strings going from the stack of $N_{c}$ branes to the magnetised one are the elementary chiral fields $Q$. The anti-chiral fields $\tilde{Q}$ have their end-points in the stack of $N_{c}$ branes and the orientifold image of the magnetised brane, whereas the fields $\rho$ with endpoints in both images of the magnetised brane are singlets under the non-abelian group but charged under the $U(1)$. The effective field theory in the electric phase $\left(N_{F}<N_{c}\right)$ was studied in detail in [6].

Here we will consider the magnetic phase appropriate for $N_{c}<N_{F}<3 N_{c} / 2$ for which instead of $Q, \tilde{Q}$ the fundamental fields are the dual fields which we denote $q, p$ and the meson-like field $\Phi$. The singlet field $\rho$ will play the role of the mass parameter. Since generically the $U(1)$ is anomalous, the modulus $T$ whose real part is the inverse gauge coupling is also charged under the $U(1)$. The $U(1)$ charges are given in the table.

\begin{tabular}{c|c|c|c|c|c} 
& $q$ & $p$ & $\rho$ & $\Phi$ & $e^{a T}$ \\
\hline$U(1)$ & $-1 / 2$ & $-1 / 2$ & -1 & 2 & 1
\end{tabular}

The effective field theory will be determined by the superpotential $W$, the gauge kinetic function $f$ and Kähler potential $K$ as follows: the gauge kinetic function for the gauge fields on the relevant D7 brane is at leading order $f=T_{s}$, where $T_{s}$ is the Kähler modulus whose real part is $\tau_{s}$. The superpotential is of the form

$$
W=W_{0}+W_{\mathrm{np}}
$$


with $W_{0}$ a flux induced superpotential which is a constant after fixing the complex structure moduli and the non-perturbative superpotential is taken to be of the moduli dependent ISS form ${ }^{1}$ :

$$
W_{\mathrm{np}}=\alpha e^{-a T_{s}}\left(\frac{p \Phi q}{\mu}+\Phi \rho\right),
$$

Here $\alpha, a, \mu$ are constants. The Kähler potential is

$$
K=-2 \log (\mathcal{V}+\xi)+K_{\text {matter }}
$$

where $\mathcal{V}$ is the volume written in terms of the Kähler moduli $\tau_{a}$ and $\xi$ corresponds to the leading order $\alpha^{\prime}$-corrections. The chiral matter Kähler potential is only known in a small field expansion as a function of the Kähler moduli, with the complex structure dependence unknown, however this is enough for our purposes here. From the analysis in [24] we can write:

$$
K_{\text {matter }}=\frac{\tau_{s}^{n}}{\mathcal{V}^{2 / 3}}\left(|\Phi|^{2}+|\rho|^{2}+|q|^{2}+|p|^{2}\right),
$$

where $n$ is the modular weight. We can then ask how the structure of the vacuum for the ISS scenario is modified once the moduli dependence of the superpotential and Kähler potential are included.

\section{The One-modulus Case}

We first consider the simplest case of a one Kähler modulus Calabi-Yau with $T=\tau+i b$, where $\tau$ denotes the Einstein frame volume of the 4-cycle $X$ and $b=\int_{X} C_{4}$. This is similar to the setup presented by Nakayama et al. [25] and is considered as a stabilisation in the spirit of KKLT [1]. After discussing the setup, we will show why it is not possible to stabilise the Kähler moduli and brane moduli in a viable regime.

The Kähler potential takes the simple form (here the explicit dependence on $g_{s}$ and $M_{P}$ is included since they are important for the numerical estimates):

$$
K=-2 M_{P}^{2} \log \left(\mathcal{V}+\frac{\xi}{g_{s}^{3 / 2}}\right)+\frac{1}{\left(g_{s} \tau\right)^{n}}\left(|\Phi|^{2}+|\rho|^{2}+|q|^{2}+|p|^{2}\right),
$$

where we kept the modulus weight general and included the leading order $\alpha^{\prime}$-corrections. The volume simply is assumed to be $\mathcal{V}=\tau_{1}^{3 / 2}$ and is in the Einstein frame. The matter fields have mass dimension 1 . The superpotential becomes

$$
W=g_{s}^{3 / 2} M_{P}^{3} W_{0}+M_{P} g_{s}^{4 / 3} e^{-a T} \alpha\left(\frac{p \Phi q}{\mu}+\rho \Phi\right) .
$$

We can now calculate the D-term potential for the anomalous $U(1)$ with the previously discussed charge assignments. Setting $g_{s}=M_{P}=1$, for simplicity of notation, the D-term

\footnotetext{
${ }^{1}$ A full string theory derivation of this non-perturbative superpotential is not yet available (see [25] for a previous discussion of this system).
} 
potential becomes:

$$
\begin{aligned}
V_{D}=\frac{1}{2 \tau}\left(\frac{-|\rho|^{2}-\frac{1}{2}\left(|q|^{2}+|p|^{2}\right)+2|\Phi|^{2}}{\tau^{n}}+\frac{1}{a}\left(\frac{-2 \sqrt{\tau}}{3\left(\xi+\tau^{3 / 2}\right)}\right.\right. & \\
& \left.\left.-n \frac{|\rho|^{2}+|p|^{2}+|q|^{2}+|\Phi|^{2}}{2 \tau^{n+1}}\right)\right)^{2},
\end{aligned}
$$

The F-term potential is calculated from the standard supergravity formula

$$
V_{F}=e^{\frac{K}{M_{P}^{2}}}\left(D_{i} W D_{\bar{j}} \bar{W} K_{i \bar{j}}^{-1}-3 \frac{|W|^{2}}{M_{P}^{2}}\right) .
$$

with $D_{i} W=\partial_{i} W+W \partial_{i} K / M_{P}^{2}$. If we assume no implicit dependence of the matter fields on the volume, the leading order contributions to the F-term potential are given by:

$$
\begin{aligned}
e^{K} & \sim \frac{1}{\left(\tau^{3 / 2}+\xi\right)^{2}}, \\
\partial W \partial \bar{W} K^{-1} & \sim\left(\frac{4}{3} \tau^{2}-\frac{4 n \tau^{2-n}}{9}\right) a^{2} e^{-2 a \tau} \alpha^{2}\left|\frac{p \Phi q}{\mu}+\rho \Phi\right|^{2} \text { for } n>0, \\
W \partial W \partial \bar{K} K^{-1}+\text { c.c. } & \sim 4 W_{0} a \alpha \tau \operatorname{Re}\left(e^{-a T}\left(\frac{p \Phi q}{\mu}+\rho \Phi\right)\right), \\
|W|^{2} \partial K \partial \bar{K} K^{-1} & \sim\left|W_{0}\right|^{2}\left(3+\frac{3 \xi}{2 \tau^{3 / 2}}+(1-n) \frac{|p|^{2}+|q|^{2}+|\rho|^{2}+|\Phi|^{2}}{\tau^{n}}\right) .
\end{aligned}
$$

Bearing in mind that the flux parameter $W_{0}$ has to be exponentially small in KKLT-like setups, the key observation for the following discussion is the fact that we have got a hierarchy between the D-term potential and the F-term potential, simply since the F-term potential is exponentially suppressed and the D-term potential is not. Therefore the Dterm potential has to vanish almost exactly, that is it should vanish up to the exponential suppression of the F-term potential.

Because of the D-term dominance, we are required to address a completely different stabilisation procedure compared to the global field theory setup of ISS. It is actually not possible to integrate out the fields at the minimum of the global SUSY model.

The multi-field minimisation process can be simplified (as in the global case) by noting that the D-term potential arising from the $S U\left(N_{F}-N_{C}\right)$ gauge theory can be minimised by simply requiring the VEV of both type of fields $q$ and $p$ to be the same, since:

$$
V_{D}^{S U\left(N_{F}-N_{C}\right)}=\frac{g^{2}}{2} \sum_{A}\left(\operatorname{Tr} q^{\dagger} T_{A} q-\operatorname{Tr} p T_{A} p^{\dagger}\right)^{2}
$$

where $T_{A}$ denotes the generator of the fundamental representation of $S U\left(N_{F}-N_{C}\right)$. It can further be seen that the minimum corresponds when both vanish $q=p=0 .^{2}$ Since the $\mathrm{D}$-term is dominant the remaining matter fields are related by a condition of the form $|\rho|^{2} \sim|\Phi|^{2}-b$, where $b$ is determined by the leading order FI term. This reduces the

\footnotetext{
${ }^{2}$ See Appendix $\mathrm{C}$ for a detailed discussion.
} 
potential as a function of, say, $|\Phi|$ and $\tau$. As expected we do not find a minimum for large values of $\tau$ where the potential shows the standard runaway behaviour as illustrated in the figure 11 below.

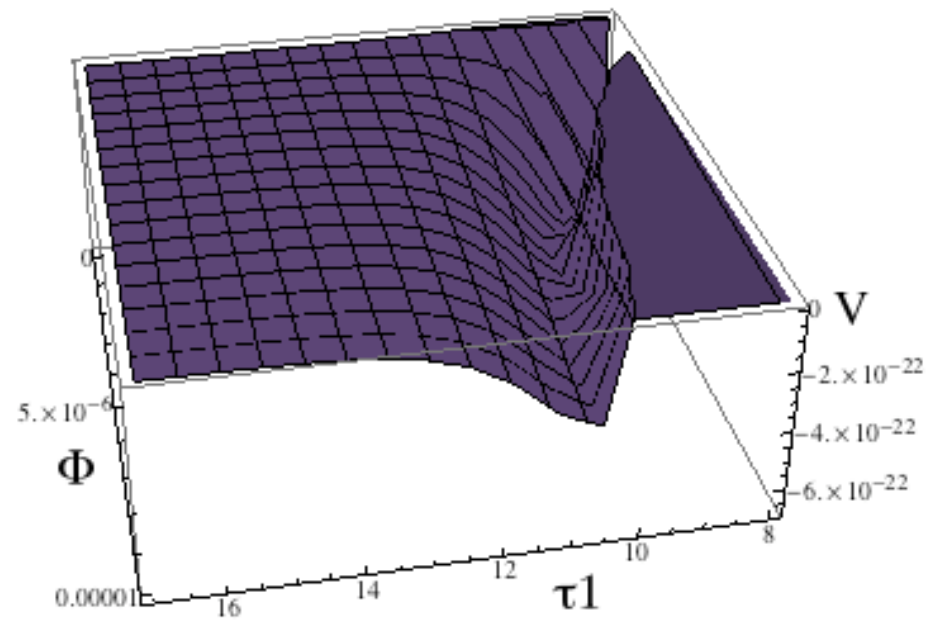

Figure 1: This plot shows the F-term potential in dependence of the Kähler modulus $\tau$ and the remaining matter degree of free$\operatorname{dom} \Phi$. We display small values of $\Phi$ in order to show the reader the runaway behaviour, which is clearly shown in the picture. Keeping $\Phi$ constant would give the typical KKLT situation.

For small values of $\tau$ local minima can be found, but in a regime that does not justify keeping only the leading order corrections to $K[25]$.

\section{The Two-moduli Case}

Since the one-modulus case leads naturally to the runaway behaviour, it is tempting to conclude that string theory realisations of the ISS mechanism do not have a metastable state but a runaway potential. We will now explore the Swiss cheese, two-moduli case which has proven to lead to qualitatively different vacuum structure in the LARGE volume scenario of moduli stabilisation and reconsider moduli stabilisation in our set-up.

We will then consider the geometry of the Calabi-Yau defined as the surface in projective space $\mathbb{P}_{[1,1,1,6,9]}$ where the volume is given by

$$
\mathcal{V}=\tau_{1}^{3 / 2}-\tau_{2}^{3 / 2}
$$

where $\tau_{1}$ denotes the large 4 -cycle and $\tau_{2}$ the small one. Then our stringy setup is given by:

$$
\begin{aligned}
& K=-2 M_{P}^{2} \log \left(\tau_{1}^{3 / 2}-\tau_{2}^{3 / 2}+\frac{\xi}{g_{s}^{3 / 2}}\right)+\frac{g_{s}^{n} \tau_{2}^{n}}{g_{s} \tau_{1}}\left(|p|^{2}+|q|^{2}+|\rho|^{2}+|\Phi|^{2}\right), \\
& W=M_{P}^{3} g_{s}^{3 / 2} W_{0}+\alpha M_{P} g_{s}^{1 / 2+n} e^{-a T_{2}}\left(\frac{p \Phi q}{\mu}+\Phi \rho\right) .
\end{aligned}
$$

In the following we will use the charge assignment

\begin{tabular}{c|c|c|c|c|c} 
& $q$ & $p$ & $\rho$ & $\Phi$ & $e^{a T_{2}}$ \\
\hline$U(1)$ & 1 & 1 & 2 & -1 & 1
\end{tabular}


That is the D7 branes are wrapping the small four-cycle and then it is $T_{2}$ that gets charged under $U(1)$ and not $T_{1}$. Then the D-term potential becomes

$$
\begin{aligned}
V_{D}=\frac{g_{s}}{2 \tau_{2}}\left[\frac{2|\rho|^{2} \tau_{2}^{n}}{g_{s}^{1-n} \tau_{1}}+\frac{\left(|q|^{2}+|p|^{2}\right) \tau_{2}^{n}}{g_{s}^{1-n} \tau_{1}}-\frac{|\Phi|^{2} \tau_{2}^{n}}{g_{s}^{1-n} \tau_{1}}+\right. \\
\left.+\frac{1}{a}\left(\frac{3 M_{P}^{2} \sqrt{\tau_{2}}}{2\left(\mathcal{V}+\frac{\xi}{g_{s}^{3 / 2}}\right)}+\frac{\left(|\rho|^{2}+|p|^{2}+|q|^{2}+|\Phi|^{2}\right) n \tau_{2}^{n-1}}{2 g_{s}^{1-n} \tau_{1}}\right)\right]^{2}
\end{aligned}
$$

where the overall $g_{s}$ arises due to the transformation from string to Einstein frame. In general there are two possible charge assignments; one is displayed above, and the other interchanges the charges $\rho$ and $\Phi$, meaning that both fields are changing their role in the D-term stabilisation.

The F-term potential is given by the usual supergravity formula (divided by $g_{s}^{2}$ which arises by integrating out the dilaton dependence)

$$
\begin{aligned}
V_{F} & =\frac{1}{g_{s}^{2}} e^{\frac{K}{M_{P}^{2}}}\left(D_{i} W D_{\bar{j}} \bar{W} K^{i \bar{j}}-3 \frac{|W|^{2}}{M_{P}^{2}}\right) \\
& =\frac{e^{\frac{K}{M_{P}^{2}}}}{g_{s}^{2}}\left(\partial_{i} W \partial_{\bar{j}} \bar{W} K^{i \bar{j}}+\partial_{i} W \partial_{\bar{j}} \bar{K} K^{i \bar{j}} \frac{\bar{W}}{M_{P}^{2}}+\frac{W}{M_{P}^{2}} \partial_{i} K \partial_{\bar{j}} \bar{W} K^{i \bar{j}}+\frac{|W|^{2}}{M_{P}^{4}} \partial_{i} K \partial_{\bar{j}} \bar{K} K^{i \bar{j}}-3 \frac{|W|^{2}}{M_{P}^{2}}\right)
\end{aligned}
$$

where the indices run over the two Kähler moduli $T_{1}, T_{2}$ and matter fields $p, q, \Phi$.

\subsection{Strategy}

Our aim is now to find a minimum of the whole potential at large volume to guarantee stability towards unknown higher order corrections. Therefore we should always keep track of the power dependence in $\tau_{1}$ which corresponds directly to the power suppression by the volume. The results presented are only reliable up to some order in the volume suppression.

Let us start by emphasising the following points: We can see that the D-term potential is suppressed by $1 / \tau_{1}^{2}$ whereas the F-term potential is at least suppressed by $1 / \tau_{1}^{3}$. This directly implies that the D-term contribution is a priori leading compared to the F-term potential and hence the global SUSY analysis of ISS will be modified. Also, minimisation with respect to the matter fields will lead to a moduli dependence of their VEVs.

Minimising the whole potential analytically is not possible due to the complexity of the system corresponding to a potential as a function of six complex fields. Instead, we will use the suppression with respect to the volume of every single term as a natural order criterion, since this allows us to discard various higher order contributions. Our approach can be summarised as follows:

1. The first step of our minimisation procedure will be to find the dependence of the matter fields (e.g. $\chi$ ) on the large Kähler modulus, at leading order, writing $\chi=\tilde{\chi} / \mathcal{V}^{m}$ and determine $m$ for each matter field $\chi$, still not fixing the matter fields completely (leaving $\tilde{\chi}$ still unfixed). 
2. After that we can minimise the matter fields completely by fixing $\tilde{\chi}$. We then end up with a scenario which looks roughly like the original LARGE volume scenario with an additional uplifting D-term.

3. We finally stabilise the potential with respect to the Kähler moduli numerically.

\subsection{Matter field stabilisation}

Similar to the one-field case, the fields $p$ and $q$ can be stabilised at $p=q=0 .{ }^{3}$ Then we need to determine $\Phi$ and $\rho$ as functions of the volume. We should note at this point that it is not possible to set any of these two fields to zero, in particular $\rho \neq 0$, since then the contribution from $\partial W \partial K K^{-1}$ vanishes as well, and we remain with only positive definite terms in the F-term potential.

We would now like to fix the implicit dependence of the matter fields on the Kähler moduli. In order to ensure that there will not be any further leading order correction to this assumption it is necessary to satisfy one of the following conditions:

After fixing the quark and antiquark fields the D-term potential looks like:

$$
V_{D}=\frac{g_{s}}{2 \tau_{2} \mathcal{V}^{4 / 3}}\left(\frac{|\rho|^{2}\left(36 a \tau_{2}+n\right)}{18 a g_{s}^{1-n} \tau_{2}^{1-n}}-\frac{|\Phi|^{2}\left(18 a \tau_{2}-n\right)}{18 a g_{s}^{1-n} \tau_{2}^{1-n}}+\frac{3 M_{P}^{2} \sqrt{\tau_{2}}}{2 a \mathcal{V}^{1 / 3}} \frac{1}{1+\frac{\xi}{g_{s}^{3 / 2} \mathcal{V}}}\right)^{2}
$$

Starting from the observation that the only negative contribution in the D-term potential comes from the term including $\Phi$ fields, it is natural to think that this term will cancel the leading order FI contribution. ${ }^{4}$ Cancelling the leading order FI-term with the $\Phi$ field implies the following implicit volume dependence:

$$
|\Phi|^{2}=\frac{|\tilde{\Phi}|^{2}}{\mathcal{V}^{1 / 3}}
$$

With this assumption we can rewrite the D-term as follows:

$$
\begin{aligned}
V_{D} & =\frac{g_{s}}{2 \tau_{2} \mathcal{V}^{4 / 3}}\left(\left(\frac{|\rho|^{2}\left(36 a \tau_{2}+n\right)}{18 a g_{s}^{1-n} \tau_{2}^{1-n}}\right)^{2}+\frac{1}{\mathcal{V}^{2 / 3}}\left(\frac{3 \sqrt{\tau_{2}}}{2 a} \frac{M_{P}^{2}}{1+\frac{\xi}{g_{s}^{3 / 2} \mathcal{V}}}-\frac{|\tilde{\Phi}|^{2}\left(18 a \tau_{2}-n\right)}{18 a g_{s}^{1-n} \tau_{2}^{1-n}}\right)^{2}\right. \\
& \left.+\frac{2}{\mathcal{V}^{1 / 3}}\left(\frac{3 \sqrt{\tau_{2}}}{2 a} \frac{M_{P}^{2}}{1+\frac{\xi}{g_{s}^{3 / 2} \mathcal{V}}}-\frac{|\tilde{\Phi}|^{2}\left(18 a \tau_{2}-n\right)}{18 a g_{s}^{1-n} \tau_{2}^{1-n}}\right)\left(\frac{|\rho|^{2}\left(36 a \tau_{2}+n\right)}{18 a g_{s}^{1-n} \tau_{2}^{1-n}}\right)\right)
\end{aligned}
$$

To cancel the FI-term we find:

$$
|\Phi|^{2}=\frac{|\tilde{\Phi}|^{2}}{\mathcal{V}^{1 / 3}}=\frac{54 M_{P}^{2} g_{s}^{1-n} \tau_{2}^{3 / 2-n}}{2\left(18 a \tau_{2}-n\right) \mathcal{V}^{1 / 3}} \stackrel{a \tau_{2} \gg 1}{=} \frac{3 M_{P}^{2} g_{s}^{1-n} \tau_{2}^{1 / 2-n}}{2 a \mathcal{V}^{1 / 3}} .
$$

This assignment minimises the potential with respect to $\Phi$ at leading order. Higher order corrections are in principle of importance if we stabilise $\rho$ at a higher order compared to

\footnotetext{
${ }^{3}$ See Appendix $\mathrm{C}$ for a detailed discussion.

${ }^{4}$ The next to leading order FI contribution is of sub-leading order even compared to the leading order F-term potential.
} 
$\Phi$. However, we show in the appendix that we can neglect these higher order corrections at our minimum.

Focusing on the mass field $\rho$, the first guess might be that there should be no difference in the implicit dependence between $\Phi$ and $\rho$ since they occur completely symmetrically after setting the quark fields to zero apart from their appearance in the D-term. In this case, we end up with the following two possibilities:

1. $\Phi$ and $\rho$ both get VEVs that are of the same order as the FI contribution but we have to cancel the D-term to leading order, so we do have to cancel the FI-term with $\Phi$. This fixes one of the matter fields up to a phase. We then have to minimise the Fterm potential on its own but with the following constraint $|\rho|^{2} \sim|\Phi|^{2}-b$, where $b$ is determined by the leading order FI part. The structure of the F-term looks promising since we find the exact same volume suppression as in the LARGE volume scenario and we can minimise with respect to the volume. But when we then try to stabilise the remaining matter field, it turns out that one cannot achieve it at large values for the volume. A detailed proof of this statement can be found in appendix A.

2. $\Phi$ and $\rho$ get VEVs that are larger then the FI-term and they then differ at that "subleading" order. The problem now is that the matter fields become so massive that we cannot trust our expansion anymore since we have to consider trans-Planckian dynamics. In addition, one runs into similar problems as above.

Since the D-term potential should be suppressed higher than the F-term potential we would like $\rho$ to be higher suppressed than $\Phi$. Hence we have to give $\rho$ a higher implicit suppression with respect to the Kähler moduli than to $\Phi$. To find the implicit suppression and have a maningful approximation, we demand that we have a very large volume and then, after the full stabilisation process is finished, check if the assumption is consistent. This gives us which term should be leading order in $\tau_{1}$, namely:

$$
\partial W \partial \bar{W} K^{-1} e^{K}
$$

Since the next to leading order contribution from the FI-term is clearly subleading, we have to suppress the term proportional to $\rho$ at least more than the leading order F-term potential term. This constraint implies that the leading order F-term contribution from the term above is independent of $\rho$. Clearly $\rho$ will occur in the leading order term coming from $\partial W \partial \bar{K} K^{-1} e^{K}$. With this implication we can determine the leading order F-term potential which looks as follows:

$$
\begin{aligned}
V_{F}=\frac{M_{P}^{4}}{g_{s}^{2} \tau_{1}^{3}}\left(\frac{g_{s}^{2+n}|\tilde{\Phi}|^{2} \alpha^{2} \sqrt{\tau_{1}} e^{-2 a \tau_{2}}}{M_{P}^{2} \tau_{2}^{n}}+\right. & \frac{4 a g_{s}^{2+n} W_{0} \alpha \tau_{2} e^{-a \tau_{2}} \operatorname{Re}(\tilde{\rho} \tilde{\Phi})}{M_{P}^{2} \tau_{1}^{1 / 4+m}} \\
& \left.+\frac{3 W_{0}^{2}\left(\frac{\xi}{g_{s}^{3 / 2}}+\frac{2 n \tau_{2}^{n}|\tilde{\Phi}|^{2}}{g_{s}^{1-n} M_{P}^{2}}\left(1-\frac{\xi}{g_{s}^{3 / 2} \tau_{2}^{3 / 2}}\right)\right)}{2 \tau_{1}^{3 / 2}}\right),
\end{aligned}
$$


where we kept the explicit dependence on $\tilde{\Phi}$ and $m$ reflects the uncertainty in the implicit suppression of $\tilde{\rho}$ with respect to the large Kähler modulus and worked in the limit $a \tau_{2} \gg 1$ for clarity. Terms including higher order corrections in $\Phi$ have not been written down but would not enter at leading order. From the structure above, we can see that we cannot stabilise the potential with respect to $\tilde{\rho}$ with a vanishing D-term since the potential gives a runaway behaviour to leading order with respect to $\tilde{\rho}$.

In order to avoid any further implicit dependence on $\tau_{1}$, we have to choose the implicit dependence in a way that both terms, the D-term contribution and leading order F-term contribution from $\partial W \partial \bar{K} K^{-1} e^{K}$ have the same $\tau_{1}$ suppression. Fortunately this can be achieved by the following implicit dependence:

$$
|\rho|^{2}=\frac{|\tilde{\rho}|^{2}}{\tau_{1}^{5 / 6}}=\frac{|\tilde{\rho}|^{2}}{\mathcal{V}^{5 / 9}} .
$$

At leading order we can stabilise with respect to $\tilde{\rho}$ as follows: Neglecting higher order corrections in $\Phi$, we face the following leading order potential in the limit $a \tau_{2} \gg 1$ evaluated at $\tilde{\Phi}=\tilde{\Phi}_{\min }$ :

$$
\begin{aligned}
V & =V_{D}+V_{F} \\
& =\frac{2 \tau_{2}^{2 n-1}|\tilde{\rho}|^{4}}{g_{s}^{1-2 n} \mathcal{V}^{22 / 9}}+\frac{3 M_{P}^{4} g_{s} \alpha^{2} e^{-2 a \tau_{2}}}{2 a \tau_{2}^{2 n-1 / 2} \mathcal{V}^{15 / 9}} \\
& +\frac{2 \sqrt{6} g_{s}^{1 / 2+n / 2} M_{P}^{3} W_{0} \alpha \sqrt{6} \tau_{2}^{9 / 4-5 n / 6} e^{-a \tau_{2}} \operatorname{Re} \tilde{\rho}}{\mathcal{V}^{22 / 9}}+\frac{3 g_{s} M_{P}^{4} W_{0}^{2}}{2 g_{s}^{3 / 2} \mathcal{V}^{27 / 9}}\left(\xi+\frac{g_{s}^{3 / 2} n \sqrt{\tau_{2}}}{a}\right)
\end{aligned}
$$

The stabilisation with respect to $\tilde{\rho}$ goes as follows: Assuming $W_{0}$ to be real, we first observe that all coefficients are in front of $\tilde{\rho}$ are real. Differentiating with respect to $\tilde{\rho}$ and its complex conjugate then directly leads to the fact that $\tilde{\rho}$ has to be real. We can now straightforwardly stabilise $\tilde{\rho}$

$$
0=\frac{\partial}{\partial \tilde{\rho}} A(\tilde{\rho})^{2}\left(\tilde{\rho}^{*}\right)^{2}+B\left(\tilde{\rho}+\tilde{\rho}^{*}\right)
$$

where $A$ and $B$ are abbreviations for the coefficients in the leading order Kähler potential. That constraint implies

$$
0=2 A \tilde{\rho}\left(\tilde{\rho}^{*}\right)^{2}+B \stackrel{\tilde{\rho} \in \mathbb{R}}{=} 2 A \tilde{\rho}^{3}+B .
$$

This can be solved directly for $\tilde{\rho}$ and we obtain in the limit $a \tau_{2} \gg 1$ :

$$
\tilde{\rho}_{\text {min }}=\left(\frac{3 a}{2}\right)^{1 / 6} M_{P} g_{s}^{1 / 2-n / 2}\left(-W_{0} \alpha\right)^{1 / 3} \tau_{2}^{3 / 4-5 n / 6} e^{-a \tau_{2} / 3} .
$$

After stabilising $\tilde{\rho}$, we end up schematically with the following leading order potential (where we fixed $n=1 / 3$ for simplicity):

$$
\begin{aligned}
V & =g_{s} M_{P}^{4}\left(\frac{3 \alpha^{2} e^{-2 a \tau_{2}}}{2 a \tau_{2}^{1 / 6} \mathcal{V}^{15 / 9}}+\frac{2\left(g_{s}^{1 / 3}|\tilde{\rho}|^{4}+\sqrt{6 a} M_{P}^{3} W_{0} \alpha \operatorname{Re}(\tilde{\rho}) \tau_{2}^{17 / 12} e^{-a \tau_{2}}\right)}{g_{s}^{5 / 3} M_{P}^{4} \tau_{2}^{1 / 3} \mathcal{V}^{22 / 9}}+\frac{3 W_{0}^{2} \xi}{2 g_{s}^{3 / 2} \mathcal{V}^{27 / 9}}\right) \\
& =g_{s} M_{P}^{4}\left(\frac{A e^{-2 a \tau_{2}}}{\tau_{2}^{1 / 6} \mathcal{V}^{15 / 9}}-\frac{B \tau_{2}^{14 / 9} e^{-4 / 3 a \tau_{2}}}{\mathcal{V}^{22 / 9}}+\frac{C}{\mathcal{V}^{27 / 9}}\right) .
\end{aligned}
$$


Where we have defined:

$$
A=\frac{3 \alpha^{2}}{2 a}, \quad B=6^{2 / 3}\left(1-\left(\frac{1}{2}\right)^{1 / 3}\right) a^{2 / 3}\left(-W_{0} \alpha\right)^{4 / 3}, \quad C=\frac{3 W_{0}^{2} \xi}{2 g_{s}^{3 / 2}} .
$$

Due to the minimisation with respect to $\rho$ we obtain a different exponential suppression than the standard LARGE volume scenario, but keep a similar structure with similar results.

We can clearly see from the potential that the extremisation with respect to $\tilde{\rho}$ gives a minimum since the coefficients $A$ and $B$ are positive and at the minimum we have a negative contribution from this factor.

\subsection{Stabilising the Kähler moduli}

To compare our stabilisation procedure to the LARGE volume scenario we go one step back to the potential before extremising with respect to $\tilde{\rho}$ :

$$
\begin{aligned}
V & =\frac{2 \tau_{2}^{2 n-1}|\tilde{\rho}|^{4}}{g_{s}^{1 / 3} \mathcal{V}^{22 / 9}}+\frac{3 g_{s} M_{P}^{4} \alpha^{2} e^{-2 a \tau_{2}}}{2 a \tau_{2}^{2 n-1 / 2} \mathcal{V}^{15 / 9}} \\
& +\frac{2 \sqrt{6} M_{P}^{3} g_{s}^{2 / 3} W_{0} \alpha \sqrt{6} \tau_{2}^{5 / 4-n / 2} e^{-a \tau_{2}} \operatorname{Re} \tilde{\rho}}{\mathcal{V}^{22 / 9}}+\frac{3 M_{P}^{4} W_{0}^{2}}{2 \mathcal{V}^{27 / 9}}\left(\frac{\xi}{g_{s}^{3 / 2}}+\frac{n \sqrt{\tau_{2}}}{a}\right)
\end{aligned}
$$

This leading order structure looks very promising since it is very close to the original LARGE volume scenario with an additional uplifting term:

$$
V_{\mathrm{LV}}=g_{s} M_{P}^{4}\left(\frac{a^{2} A^{2} \sqrt{\tau_{2}} e^{-2 a \tau_{2}}}{\mathcal{V}}+\frac{W_{0} A a \tau_{2} e^{-a \tau_{2}}}{\mathcal{V}^{2}}+\frac{W_{0}^{2} \xi}{\mathcal{V}^{3}}+\frac{V_{\mathrm{uplift}}}{\mathcal{V}^{2}}\right)
$$

where we neglected numerical constants for clarity. The different suppressions with respect to the volume are reflected in an overall rescaling. Nevertheless, there is a slight difference in the power dependence with regard to the volume in the $\partial W \partial \bar{K} K^{-1} e^{K}$ (21/9 would be perfect) which spoils the possibility of an exact analytical discussion of the minimisation as it was possible in the LARGE volume case. However the difference to the original setup is rather small. Therefore the suppression looks to be a bit larger driving the minimum to larger values. Obviously the adhoc uplifting contribution from previous LARGE volume constructions gets replaced by a concrete supersymmetric D-term contribution.

After stabilising the matter fields, we now have a potential which only depends on the Kähler moduli. Since we are not able to calculate the vacuum structure analytically we find the minima numerically. To be more explicit we will specialise to the modulus weight $n=1 / 3$ from now on, which is for practical reasons only since similar results will hold for other weights. After stabilising the matter fields we obtained the leading order potential in the limit $a \tau_{2} \gg 1$ given by (4.16), which can be written in terms of $\tau_{1,2}$ as:

$$
V=g_{s} M_{P}^{4}\left(\frac{A e^{-2 a \tau_{2}}}{\tau_{1}^{5 / 2} \tau_{2}^{1 / 6}}-\frac{B e^{-4 / 3 a \tau_{2}} \tau_{2}^{14 / 9}}{\tau_{1}^{11 / 3}}+\frac{C}{\tau_{1}^{9 / 2}}\right)
$$


After fixing the matter fields to their value at the minimum, the uplifting term from the D-term seems to disappear since we only have three terms in the potential which are similar to the original LARGE volume. However the effect of the D-term potential can be seen in the change of the exponential suppression of the term proportional to $B$ which corresponds to the negative contribution. This means that we have a smaller negative contribution compared to the setup without the D-term. Despite the fact that the D-term does not seem to be present anymore, it affects the shape of the potential in exactly the way an uplifting term does.

In general, the shape of the potential after fixing the matter fields suggests that there is a LARGE volume like structure with possible D-term lifting. Working strictly within the Kähler cone (i.e. $\tau_{2} \ll \tau_{1}$ ), at relatively small values of the volume the term proportional to the $\alpha^{\prime}$ corrections is dominant. In an intermediate range the attenuated negative contribution proportional to $W_{0}$ can be dominant and at large volumes the leading order contribution will come from the $\partial W \partial \bar{W} K^{-1}$ part. Due to the domination of this at large volumes, the potential approaches zero from above in all directions. It is in fact straigtforward to see that when the volume tends to infinity, the negative term cannot dominate.

This behaviour at large volume is in agreement with the uplifted LARGE volume scenario, where we approach zero from above. Therefore, by changing the value of the parameters we may obtain an AdS, almost flat or dS minimum or have a runaway behaviour. This is similar to the lifted LARGE volume scenario but different from the unlifted one where zero is approached from below, so we can see the indirect effect of the D-term to uplift the minimum. Notice also that it is different from the previous ways of getting de Sitter space from a purely supersymmetric potential [26], [27], [28] in which the stabilised value of the volume was relatively small, whereas here we are obtaining exponentially large volumes.

Let us study an illustrative example of these possible vacuum structures: First of all, we are indeed able to stabilise the Kähler moduli at large values for the large Kähler modulus $\tau_{1}$. For instance as depicted in figure 2, we are able to stabilise the Kähler moduli with the following naturally chosen parameters:

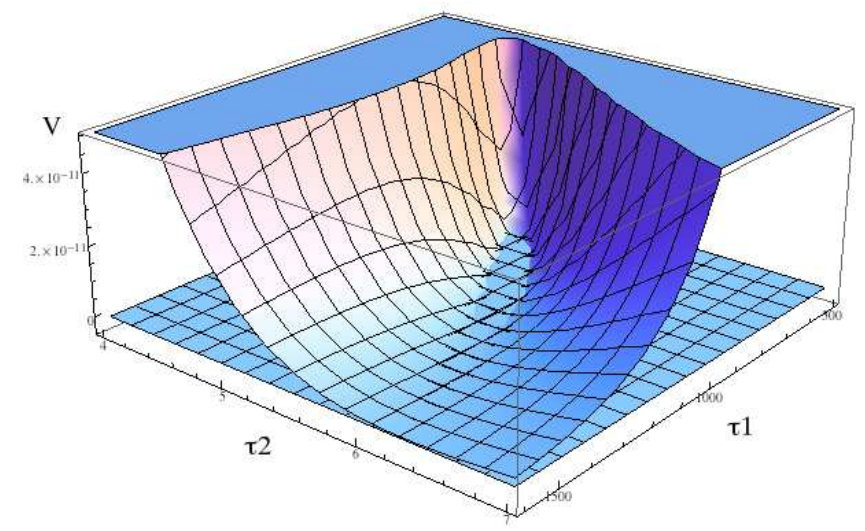

Figure 2: A plot of the numerical behaviour of the scalar potential with respect to the Kähler moduli showing an AdS minimum.

\begin{tabular}{c|c|c|c|c|c|c|c|c|c}
$\tau_{1}$ & $\tau_{2}$ & $V_{\min }$ & $\Phi$ & $\rho$ & $W_{0}$ & $a$ & $g_{s}$ & $\alpha$ & $\xi$ \\
\hline 768.03 & 5.20 & $-3.03 \times 10^{-12} g_{s} M_{P}^{4}$ & $0.34 g_{s}^{1 / 3} M_{P}$ & $0.12 g_{s}^{1 / 3} M_{P}$ & -15.85 & 0.6 & 0.1 & 1.10 & 0.32
\end{tabular}


Table 1: Showing the parameter values in the AdS-example.

In addition to the usual stabilisation corresponding to an AdS geometry, we now can achieve a stabilisation of the Kähler moduli that correspond to a dS geometry. Taking the previous example, this is achieved by tuning the flux parameter from $W_{0}=-15.85$ to $W_{0}=-15.25$. The resulting figure is 3 and the numerical values in this example are summarised in the following table:



Figure 3: A plot of the numerical behaviour of the scalar potential with respect to the Kähler moduli showing a dS minimum.

\begin{tabular}{c|c|c|c|c|c|c|c|c|c}
$\tau_{1}$ & $\tau_{2}$ & $V_{\min }$ & $\Phi$ & $\rho$ & $W_{0}$ & $a$ & $g_{s}$ & $\alpha$ & $\xi$ \\
\hline 873.40 & 5.44 & $1.83 \times 10^{-13} g_{s} M_{P}^{4}$ & $0.33 g_{s}^{1 / 3} M_{P}$ & $0.11 g_{s}^{1 / 3} M_{P}$ & -15.25 & 0.6 & 0.1 & 1.10 & 0.32
\end{tabular}

Table 2: Showing the parameter values in the dS-example.

Changing the parameters too drastically now results in a disappearance of the minimum as depicted in figure 1 .

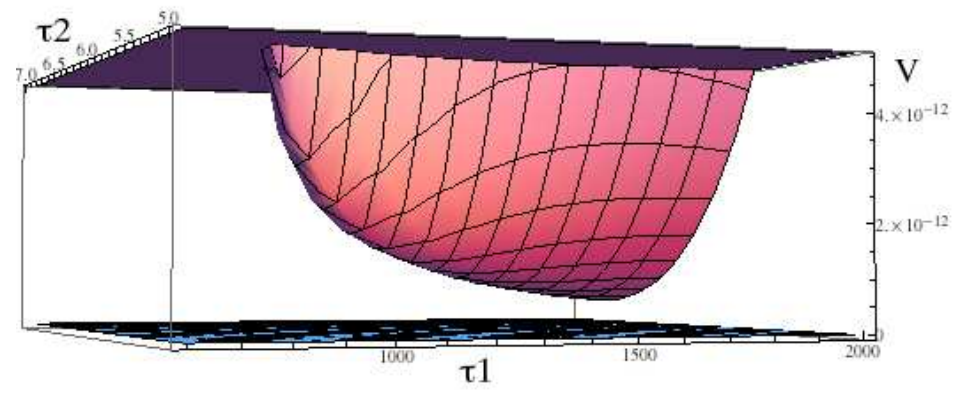

Figure 4: A plot of the numerical behaviour of the scalar potential with respect to the Kähler moduli showing no minimum.

In general we find a smooth transition from AdS to dS solutions. We may tune the parameters to obtain almost Minkowski minima ${ }^{5}$. The following example 5 shows a considerable tuning to a minimal value for the potential of $V=5.74 \times 10^{-26}$.

\footnotetext{
${ }^{5}$ In principle further tuning freedom can be achieved if the magnetised D7 brane is considered to be in a warped region [2] as in the original proposal of [1] for the lifting anti-D3 brane.
} 


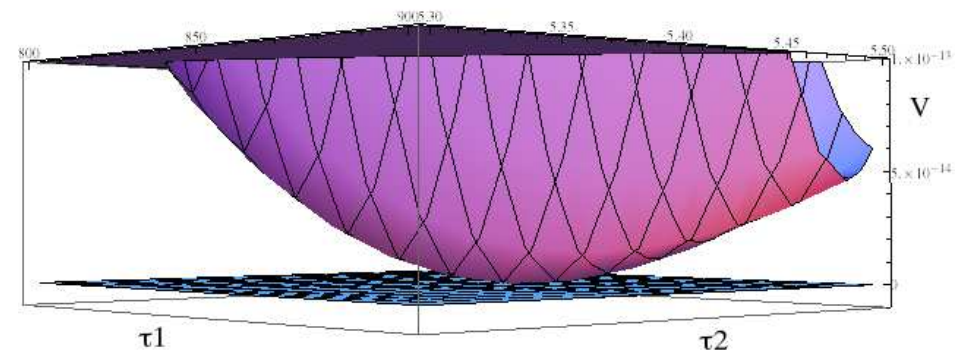

Figure 5: A plot of the numerical behaviour of the scalar potential with respect to the Kähler moduli with a minimum of $V=5.74 \times 10^{-26}$.

For a full analysis we should check our analytical results for the matter fields by visualising the potential with respect to the matter fields as well. In the case of the dS example from the previous section we obtain the following figures 6 and 7 .



Figure 6: A plot of the numerical behaviour of the scalar potential with respect to the matter field $\rho$.

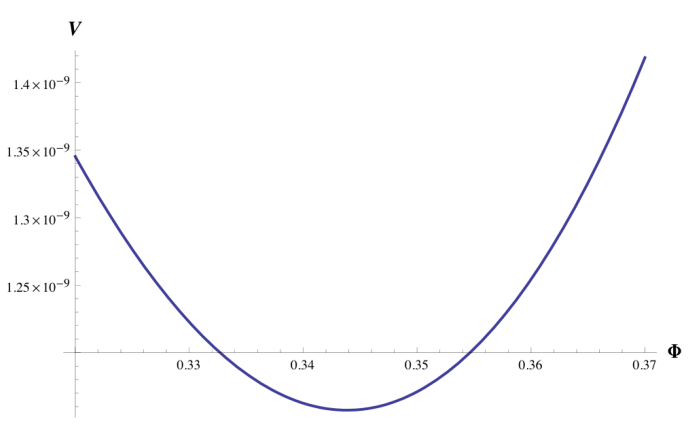

Figure 7: A plot of the numerical behaviour of the scalar potential with respect to the matter field $\Phi$.

Both plots support our previous analysis with a minimum in the predicted region. In particular the correction to $\Phi$ does not spoil our uplifting mechanism.

\section{D7 Soft-Terms}

We now would like to study the mechanism of supersymmetry breaking within the 2-moduli framework presented in the previous sections. For this we assume a brane construction on a separate four cycle $\tau_{3}$ (e.g. through an $S U(5)$ GUT model [30]) leading at low-energies to a MSSM realisation. The Standard Model cycle $\tau_{3}$ cannot be stabilised by non-perturbative effects as pointed out in [33], however loop effects could stabilise the additional cycle [39], leaving us for now with a regime of effective field theory $\left(\tau_{3} \neq 0\right)$. This ansatz is slightly different from the original study of soft-terms in the LARGE volume scenario (cf. [22] and [29]), but was recently discussed in [34]. ${ }^{6}$.

The volume in this extended Swiss-cheese Calabi-Yau can be written as $\mathcal{V}=\tau_{1}^{3 / 2}$ $\tau_{2}^{3 / 2}-\tau_{3}^{3 / 2}$. The philosophy of our approach is depicted in figure 8 below.

\footnotetext{
${ }^{6}$ We concentrate here on generic D7 brane soft terms without including explictly the mechanism for stabilising the MSSM modulus. We only assume that it is not stabilised at a singular point. A detailed study of this consideration is left for future work.
} 


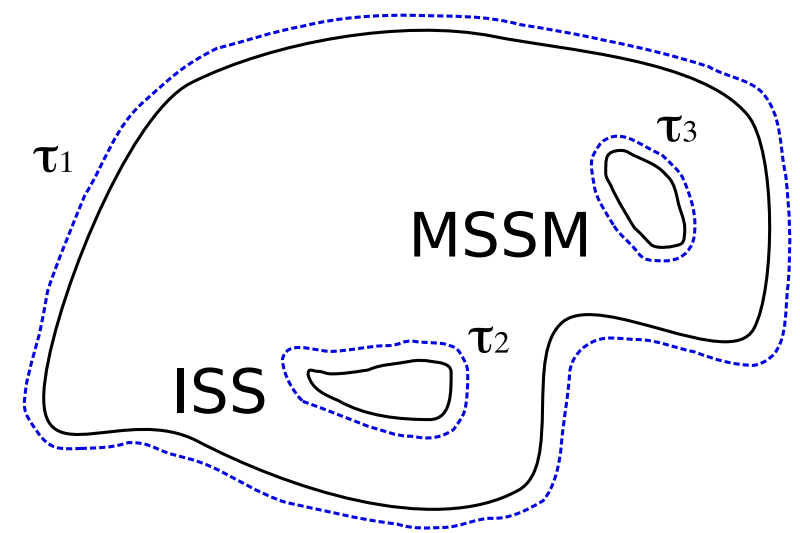

Figure 8: In the simplest model the geometry of the Calabi-Yau is parametrised by three 4 cycles. $\tau_{1}$ is the large cycle; $\tau_{2}, \tau_{3}$ denote the small (blow-up) cycles. We assume the MSSM to be localised at $\tau_{3}$ and our ISS brane setup is localised on $\tau_{2}$.

We start with the following general effective supergravity ansatz:

$$
\begin{aligned}
& K=-2 M_{P}^{2} \log \left(\mathcal{V}+\xi s^{3 / 2}\right)-\log (s)+\frac{\tau_{2}^{n}}{\tau_{1}}\left(|p|^{2}+|q|^{2}+|\rho|^{2}+|\Phi|^{2}\right)+\tilde{K}^{i}\left|v_{i}\right|^{2} \\
& W=M_{P}^{3} g_{s}^{3 / 2} W_{0}+M_{P} g_{s}^{1 / 2+n} \alpha e^{-a T_{2}}\left(\frac{p \Phi q}{\mu}+\rho \Phi\right)+W_{\mathrm{MSSM}}
\end{aligned}
$$

where we reintroduced the dilaton dependence $s ; v$ denotes one of the chiral superfields within the MSSM whose superpotential is given by $W_{\text {MSSM }}$. For now, the correct form of the moduli weights in the Kähler potential is not of major importance, and we keep our study as general as possible.

A priori we can say that the scheme of supersymmetry breaking is gravity (moduli) mediated. The amount of gauge mediated contribution can be seen in the fact that the masses associated with the hidden sector matter fields $\rho$ and $\Phi$ are larger or of the order of the gravitino mass (see the appendix for an estimate of the masses associated to our model). In due course we will establish the same or even stronger contributions to the moduli mediated soft masses compared to the LVS and we therefore can neglect contributions arising from anomaly mediation.

We follow the standard mechanism of calculating soft supersymmetry breaking terms as for example used and described in [35].

\section{Gravitino and Gaugino Masses}

The gravitino mass is found to have the standard volume suppression:

$$
\begin{aligned}
m_{3 / 2} & =e^{\frac{K}{2 M_{P}^{2}} \frac{|W|}{M_{P}^{2}}} \\
& =\frac{M_{P}\left|W_{0}\right| g_{s}^{1 / 2}}{\mathcal{V}_{E}} .
\end{aligned}
$$

where the subscript $E$ is added to clarify that this is the Einstein-frame volume.

The F-terms needed for the remaining soft-terms are calculated in detail in appendix F. Gaugino masses are in general given by

$$
M_{a}=\frac{1}{2}\left(\operatorname{Re} f_{a}\right)^{-1} F^{m} \partial_{m} f_{a} .
$$


Assuming our standard model is constructed via D7 branes, the gauge kinetic function is

$$
f_{D 7}=T_{3}
$$

Using those results, we find for the D7 brane gaugino masses the following mass:

$$
M_{D 7} \sim \frac{W_{0} M_{P} g_{s}^{1 / 2}}{2 \mathcal{V}_{E}}=\frac{m_{3 / 2}}{2}
$$

Regarding the volume suppression of the gaugino masses, the results coincide with the LARGE volume analysis. The additional suppression of the gaugino masses due to a next to leading order cancellation in the F-terms (discussed in [36]) does not occur since $\tau_{3}$ is not stabilised by non-perturbative effects.

\section{Scalar Masses}

Scalar masses can be found by the following formula

$$
m_{i}^{2}=m_{3 / 2}^{2}-F^{m} \bar{F}^{\bar{n}} \partial_{m} \partial_{\bar{n}} \log \tilde{\mathcal{K}}_{i},
$$

where we neglect contributions from the vacuum energy. ${ }^{7}$ To calculate the scalar masses we have to specify the matter metric $\tilde{\mathcal{K}}_{i}$. However we can start with the following general ansatz

$$
\tilde{\mathcal{K}}_{i} \sim \frac{\tau_{3}^{b} s^{c}}{\tau_{1}^{d}} .
$$

This gives the following leading order results

$$
\begin{aligned}
\partial_{\tau_{1}} \partial_{\tau_{1}} \log \tilde{\mathcal{K}}_{i} & =\frac{d}{4 \tau_{1}^{2}}, \\
\partial_{\tau_{3}} \partial_{\tau_{3}} \log \tilde{\mathcal{K}}_{i} & =-\frac{b}{4 \tau_{3}^{2}}, \\
\partial_{s} \partial_{s} \log \tilde{\mathcal{K}}_{i} & =-\frac{c}{4 s^{2}} .
\end{aligned}
$$

Using the results for the F-terms derived in the appendix, we find the following results:

$$
\begin{aligned}
\left(F^{\tau_{1}}\right)^{2} \partial_{\tau_{1}} \partial_{\tau_{1}} \log \tilde{\mathcal{K}}_{i} & \sim \frac{d W_{0}^{2} M_{P}^{2} g_{s}}{\mathcal{V}^{2}} \\
\left(F^{\tau_{3}}\right)^{2} \partial_{\tau_{3}} \partial_{\tau_{3}} \log \tilde{\mathcal{K}}_{i} & \sim-\frac{b M_{P}^{2} W_{0}^{2} g_{s}}{\mathcal{V}^{2}} \\
\left(F^{s}\right)^{2} \partial_{s} \partial_{s} \log \tilde{\mathcal{K}}_{i} & \sim-\frac{c M_{P}^{2} W_{0}^{2} g_{s}}{\mathcal{V}^{4}}
\end{aligned}
$$

We can directly see that the contribution arising from the dilaton is negligibly small. In addition, we find that the leading order term proportional to the gravitino mass vanishes if $d-b=1$. If this relation is satisfied, we have to identify the next to leading order

\footnotetext{
${ }^{7}$ Here the addition of D-terms does not change the formula for scalar masses since the SM fields are not charged under the anomalous $U(1)$ in the hidden sector. A general formula was presented for example in [37].
} 
contribution. Unlike in the original LARGE volume analysis, there is no sub-leading cancellation in the F-terms [34] for the Kähler moduli since the $e^{-2 a \tau_{2}}$ term in (4.20) comes from $\partial_{\phi} W \partial_{\phi} W K^{\phi \phi}$ rather than $\partial_{\tau_{2}} W \partial_{\tau_{2}} W K^{\tau_{2} \tau_{2}}$ and the D-term uplifting contribution require a slightly different stabilisation as discussed in the previous section. This determines the sub-leading contribution (see appendix for further details) to come from the mixing of the non-perturbative leading order contribution to the F-term and the other leading order F-term contribution. We obtain the interesting result:

$$
\begin{aligned}
F^{\tau_{1}} F_{\mathrm{np}}^{\tau_{1}} \partial_{\tau_{1}} \partial_{\tau_{1}} \log \tilde{\mathcal{K}}_{i} \sim \frac{2 d W_{0} g_{s}^{m} \tilde{\rho} \tilde{\Phi} \tau_{2} e^{-a \tau_{2}}}{\mathcal{V}^{2} \tau_{1}^{2 / 3}} \\
F^{\tau_{3}} F_{\mathrm{np}}^{\tau_{3}} \partial_{\tau_{3}} \partial_{\tau_{3}} \log \tilde{\mathcal{K}}_{i} \sim-\frac{2 b W_{0} g_{s}^{m} \tilde{\rho} \tilde{\Phi} \tau_{2} e^{-a \tau_{2}}}{\mathcal{V}^{2} \tau_{1}^{2 / 3}}
\end{aligned}
$$

Evaluating these contributions at the LARGE volume minimum $\left(e^{a \tau_{2}} \sim \mathcal{V}^{x}\right)$ gives the following common volume suppression:

$$
F^{\tau_{i}} F_{\mathrm{np}}^{\tau_{i}} \partial_{\tau_{i}} \partial_{\tau_{i}} \log \tilde{\mathcal{K}}_{i} \sim \frac{d-b}{\mathcal{V}^{2+\frac{4}{9}+\frac{4 x}{3}}}
$$

Overall we find the general expression for the scalar masses to be

$$
m_{i}^{2}=(1-d+b) m_{3 / 2}^{2}-2(d-b) \frac{W_{0} g_{s}^{m} \tilde{\rho} \tilde{\Phi} \tau_{2} e^{-a \tau_{2}}}{\mathcal{V}^{2} \tau_{1}^{2 / 3}}
$$

where the second term is subleading since it is higher suppressed with respect to the volume.

For example, taking $d=1$ and $b=1 / 3$ as in previous sections and in the analysis of soft terms in the LVS [29], we obtain no cancellation at leading order and find the following scalar masses:

$$
m_{i}^{2}=\frac{1}{3} m_{3 / 2}^{2}+\text { higher order corrections. }
$$

\section{A-terms}

The A-terms are given by

$$
A_{i j k}=F^{m}\left(\partial_{m} K+\partial_{m} \log Y_{i j k}-\partial_{m} \log \tilde{K}_{i} \tilde{K}_{j} \tilde{K}_{k}\right)
$$

Assuming a constant $Y_{i j k}$ we can estimate the A-terms to be given by the following expression at leading order:

$$
\begin{aligned}
A_{i i i} & =-\frac{2 \tau_{1} \sqrt{g_{s}}\left|W_{0}\right| M_{P}}{\mathcal{V}}\left(\frac{3}{2 \tau_{1}}-\frac{3 d}{2 \tau_{1}}\right)-\frac{2 \tau_{3} \sqrt{g_{s}}\left|W_{0}\right| M_{P}}{\mathcal{V}}\left(\frac{3 \sqrt{\tau_{3}}}{2 \mathcal{V}}+\frac{3 b}{2 \tau_{3}}\right)+\frac{2 \tau_{2} \sqrt{g_{s}}\left|W_{0}\right| M_{P}}{\mathcal{V}}\left(\frac{3 \sqrt{\tau_{2}}}{2 \mathcal{V}}\right) \\
& =-3 m_{3 / 2}(1-d+b)+\text { h. о. },
\end{aligned}
$$

which are universal and of the order of the gravitino mass. 


\section{Short Summary}

Overall we find three different scenarios according to the value of $d-b$ and $\tau_{3}$ :

1. If $d-b \neq 1$ the soft terms are of the order the gravitino mass.

2. If $d-b=1$ the gaugino masses are of order of the gravitino mass but scalar masses and $A$-terms are much more suppressed.

In all cases the soft terms are universal $[29,38]$.

We can compare these results with the original LARGE volume analysis from [29] where cancelations in the F-terms occurred due to the minimization of the small cycles via non-perturbative effects and the soft-terms where found to be:

$$
M_{i} \sim \frac{m_{3 / 2}}{\log m_{3 / 2}}, m_{i}=n M_{i}, A_{\alpha \beta \gamma}=-3 n M_{i} .
$$

The additional small suppression of the soft-terms compared to the gravitino mass found in the original LARGE volume analysis is not present here and this feature distinguishes the structure of soft-terms from both scenarios.

The difference to the corresponding 3-cycle set-up in the LVS [34] is that the slight difference in the potential ( $D$-term uplifting contribution especially) renders the scalar masses not affected by sub-leading corrections in the F-term or the Kähler metric. A detailed quantitative analysis of this scenario is out of the scope of this article.

\section{Kähler moduli inflation}

In the previous sections we described a mechanism that allows us to obtain dS vacua within a fully $\mathcal{N}=1$ supersymmetric action. We now would like to show how to implement the model of Kähler moduli inflation [16] within this framework.

The starting point of the discussion is the modification of the original geometry of $\mathbb{P}_{[1,1,1,6,9]}$ by adding an additional small 4-cycle. The volume is modified to

$$
\mathcal{V}=\tau_{1}^{3 / 2}-\tau_{2}^{3 / 2}-\tau_{3}^{3 / 2}
$$

On that additional 4-cycle we assume a non-perturbative effect $B e^{-b T_{3}}$. The Kähler and superpotential are changed to:

$$
\begin{aligned}
& K=-2 M_{P}^{2} \log \left(\mathcal{V}+\frac{\xi}{g_{s}^{3 / 2}}\right)+\frac{g_{s}^{n} \tau_{2}^{n}}{g_{s} \tau_{1}}\left(|p|^{2}+|q|^{2}+|\rho|^{2}+|\Phi|^{2}\right), \\
& W=M_{P}^{3} g_{s}^{3 / 2} W_{0}+M_{P} g_{s}^{1 / 2+n} e^{-a T_{2}} \alpha\left(\frac{p \Phi q}{\mu}+\Phi \rho\right)+M_{P}^{3} g_{s}^{3 / 2} B e^{-b T_{3}}
\end{aligned}
$$

This change alters the leading order potential to:

$$
V=V_{\text {old }}+g_{s} M_{P}^{4}\left(\frac{8(b B)^{2} \sqrt{\tau_{3}} e^{-2 b \tau_{3}}}{3 \mathcal{V}}+\frac{4 W_{0} b B \tau_{3} e^{-b \tau_{3}}}{\mathcal{V}^{2}}\right),
$$


where $V_{\text {old }}$ is the potential discussed in previous sections. The old potential is independent of $\tau_{3}$ and we can hence stabilise the new part with respect to $\tau_{3}$ at constant volume. We obtain in the limit $b T_{3} \gg 1$ :

$$
b B e^{-b T_{3}}=\frac{W_{0} \sqrt{\tau_{3}}}{12 \mathcal{V}} .
$$

Plugging this value into the original potential gives the following contribution at the extremal value for $\tau_{3}$ :

$$
V=V_{\text {old }}-\frac{17 W_{0}^{2}\left(\tau_{3}^{0}\right)^{3 / 2}}{54 \mathcal{V}^{3}}
$$

Since the contribution from the potential including $\tau_{3}$ is negative, we have to be at a minimum with respect to $\tau_{3}$. Having a negative contribution from the inflationary potential at the minimum then also requires to stabilize the potential at a "large" positive value and not at an almost Minkowski minimum. The assumption that $\mathcal{V}$ can be taken to be constant during inflation is justified in the limit $b \gg a$ as the effect of the additional cycle $\tau_{3}$ towards the potential is negligibly small compared to the old potential. Hence we end up with the same potential as in the Kähler moduli inflation but with the advantage that we can use our new mechanism to stabilise the volume at a positive vacuum energy.

Following the analysis of the original paper [16], we can now calculate the inflationary characteristics of this model. Our inflationary potential is given by

$$
V=V_{\text {old }}+\frac{4 \tau_{3} W_{0} b B e^{-b \tau_{3}}}{\mathcal{V}^{2}}
$$

where we neglect the higher suppressed and hence irrelevant term including the double exponential. The physically relevant parameter is the canonically normalised version of $\tau_{3}$ which is found to be at leading order

$$
\tau_{3}^{c}=\sqrt{\frac{3}{4}} \frac{\tau_{3} M_{P}}{\left(\tau_{1}^{0}\right)^{3 / 4}\left(\tau_{3}^{0}\right)^{1 / 4}},
$$

where the exponent "0" denotes that the value is taken at the minimum. Rewriting the potential in terms of the canonically normalised field $\tau_{3}^{c}$ shows the exponential suppression with respect to the volume

$$
V=V_{0}+\frac{4 W_{0} b B}{M_{P} \mathcal{V}^{2}}\left(\frac{3 \mathcal{V}}{4}\right)^{2 / 3}\left(\tau_{3}^{c}\right)^{4 / 3} \exp \left[-b\left(\frac{3 \mathcal{V}}{4}\right)^{2 / 3} \frac{\left(\tau_{3}^{c}\right)^{4 / 3}}{M_{P}}\right]
$$

From this potential we find the following slow-roll parameters:

$$
\begin{aligned}
& \epsilon=\frac{M_{P}^{2}}{2}\left(\frac{V^{\prime}}{V}\right)^{2}=\frac{32\left(W_{0} b B\right)^{2}}{3 V_{0}^{2} \mathcal{V}^{4}} \tau_{3}^{1 / 2}\left(1-b \tau_{3}\right)^{2} e^{-2 b \tau_{3}} \\
& \eta=M_{P}^{2} \frac{V^{\prime \prime}}{V}=\frac{4 W_{0} b B}{3 \sqrt{\tau_{3}} V_{0} \mathcal{V}^{2}}\left(1-9 b \tau_{3}+4\left(b \tau_{3}\right)^{2}\right) e^{-b \tau_{3}} \\
& \xi=M_{P}^{4} \frac{V^{\prime} V^{\prime \prime \prime}}{V^{2}}=-\frac{32\left(W_{0} b B\right)^{2}}{9 V_{0}^{2} \mathcal{V}^{4} \tau_{3}}\left(1-b \tau_{3}\right)\left(1+11 b \tau_{3}-30\left(b \tau_{3}\right)^{2}+8\left(b \tau_{3}\right)^{3}\right) e^{-2 b \tau_{3}}
\end{aligned}
$$

where the derivatives are taken with respect to the canonically normalised fields. In these parameters the only small difference is that we have not specified $V_{0}$ yet, which will be of a 
similar form compared to the LARGE volume scenario. From the slow-roll parameters one can determine the spectral index and its running as

$$
\begin{aligned}
n-1 & =2 \eta-6 \epsilon+O(\xi), \\
\frac{d n}{d \ln k} & =16 \epsilon \eta-24 \epsilon^{2}-2 \xi .
\end{aligned}
$$

In analogy to the original discussion of Kähler moduli inflation we find the number of e-foldings to be given by

$$
N_{e}=\int_{\phi_{\text {end }}}^{\phi} \frac{V}{V^{\prime}} d \phi=\frac{3 V_{0}}{16 W_{0} b B} \int_{\tau_{3}^{\text {end }}}^{\tau_{3}} \frac{e^{b \tau_{3}}}{\sqrt{\tau_{3}}\left(1-b \tau_{3}\right)} d \tau_{3} .
$$

To match the COBE normalisation for the density fluctuations $\delta_{H}=1.92 \times 10^{-5}$ we have to satisfy the following constraint

$$
\frac{V^{3 / 2}}{M_{P}^{3} V^{\prime}}=5.2 \times 10^{-4}
$$

where the potential is evaluated at the horizon exit, which means $N_{e}=50-60$ e-foldings before the end of inflation. This endows us with a constraint to determine the contribution from the original potential $V_{0}$. In general the only modification on the model of Kähler moduli inflation is given by the fact that the old LARGE volume contribution is replaced by the new potential which was developed and discussed in the previous sections. It is therefore most likely that a concrete calculation, which we have not completed yet, will give the same numerical results as in the original Kähler moduli scenario, which are given by:

- The tensor-to-scalar ratio was found to be

$$
r \sim 16 \epsilon .
$$

- For 50 - 60 e-foldings, the model gives rise to the following characteristics:

$$
\begin{aligned}
0.960 & <n<0.967, \\
-0.0006 & <\frac{d n}{d \ln k}<-0.0008, \\
0 & <|r|<10^{-10}, \\
10^{5} l_{s}^{6} & \leq \mathcal{V} \leq 10^{7} l_{s}^{6} .
\end{aligned}
$$

\section{3-Parameter K3 fibration and Fibre Inflation}

To show the generality of the uplifting mechanism, we now consider the example of a 3parameter K3 fibration which allows Kähler moduli stabilisation at LARGE volume (cf. [39] and [19]). We start with the same expression for the volume as in the mentioned articles

$$
\mathcal{V}=\alpha \sqrt{\tau_{1}}\left(\tau_{2}-\beta \tau_{1}\right)-\gamma \tau_{3}^{3 / 2}
$$


It was shown that the combination $\alpha \sqrt{\tau_{1}}\left(\tau_{2}-\beta \tau_{1}\right)$ plays the rôle of the exponentially dominating volume as in the original LARGE volume scenario. $\tau_{3}$ plays the role of a blow-up and is crucial for the existence of a stable minimum at LARGE volume. Taking only the scalar potential, one still remains with one flat direction, corresponding essentially to $\tau_{1}$. It was shown in [39] that this runaway behaviour can be stabilised by considering loop-corrections to the potential.

To embed our uplifting scenario in this K3 setup, we need to know where (i.e. on which cycle) non-perturbative effects are required. To keep the same structure of moduli stabilisation it is necessary to place our brane setup on the blow-up cycle $\tau_{3}$. Since both of the other cycles have to be too large and the non-perturbative effects are hence negligibly small on those cycles.

Our setup in terms of the Kähler and superpotential then looks like

$$
\begin{aligned}
& K=-2 M_{P}^{2} \log \left(\mathcal{V}+\frac{\xi}{g_{s}^{3 / 2}}\right)+\frac{\tau_{3}^{m}}{g_{s}^{1-m} \mathcal{V}^{2 / 3}}\left(|p|^{2}+|q|^{2}+|\rho|^{2}+|\Phi|^{2}\right), \\
& W=g_{s}^{3 / 2} M_{P}^{3} W_{0}+M_{P} g_{s}^{1 / 2+m} \delta e^{-a T_{3}}\left(\frac{p \Phi q}{\mu}+\rho \Phi\right) .
\end{aligned}
$$

Wrapping around the 4-cycle parametrised by $\tau_{3}$ gives us the following leading order structure in the D-term potential:

$$
V_{D}=\frac{1}{2 \tau_{3}}\left(\frac{\tau_{3}^{m}}{\mathcal{V}^{2 / 3}}\left(q_{\rho}|\rho|^{2}+q_{\Phi}|\Phi|^{2}\right)+Q_{\tau_{3}}\left(\frac{3 \sqrt{\tau_{3}}}{2(\mathcal{V}+\xi)}+\frac{m \tau_{3}^{m-1}\left(|\rho|^{2}+|\Phi|^{2}\right)}{\mathcal{V}^{2 / 3}}\right)\right)^{2}
$$

where we directly set the quark fields to zero. The structure of the D-term potential is essentially the same as in the analysis of the $\mathbb{P}_{[1,1,1,6,9]}$ geometry from the previous section. This not only allows us to set the quark fields to zero but also to cancel the FI-term with the least suppressed $\Phi$-field.

Hence obtain the following implicit dependence of $\Phi$ on the large Kähler moduli

$$
|\Phi|^{2}=\frac{|\tilde{\Phi}|^{2}}{\mathcal{V}^{1 / 3}}=\frac{Q_{\tau_{3}} \mathcal{V}^{2 / 3} \tau_{3}^{\frac{1}{2}-m}}{2 q_{\Phi}(\mathcal{V}+\xi)}\left(1+\frac{m Q_{\tau_{3}}}{q_{\Phi} \tau_{3}}\right)^{-1} \approx \frac{Q_{\tau_{3}} \tau_{3}^{\frac{1}{2}-m}}{2 q_{\Phi} \mathcal{V}^{1 / 3}}
$$

As in the Swiss-cheese case we can only assume at this stage that $\rho$ is implicitly higher suppressed than $\Phi$ with respect to the Kähler moduli.

With this assumption and the knowledge from the previous study in the Swiss-cheese case we can now estimate the leading order contributions to the F-term potential:

$$
\begin{aligned}
e^{K} & \sim \frac{1}{\mathcal{V}^{2}} \\
\partial W \partial \bar{W} K^{-1} & \sim g_{s}^{2+m} M_{P}^{2} \delta^{2} e^{-2 a \tau_{3}} \frac{\mathcal{V}^{1 / 3}|\tilde{\Phi}|^{2}}{\tau_{3}^{m}} \\
\partial W \partial \bar{K} K^{-1} \bar{W}+\text { c. c. } & \sim \frac{12 W_{0} g_{s}^{3 / 2+m} M_{P}^{3} \delta \operatorname{Re} \tilde{\rho} \tilde{\Phi} e^{-a \tau_{3}} \gamma \mathcal{V}^{1 / 3} \tau_{3}\left(m+a \tau_{3}\right)}{\left(3 g_{s} M_{P}^{2} \gamma \mathcal{V}^{1 / 3} \tau_{3}^{3 / 2}+2 g_{s}^{m} m \alpha \sqrt{\tau_{1}}\left(\beta \tau_{1}-\tau_{2}\right) \frac{\tau_{3}^{m}|\tilde{\Phi}|^{2}}{\mathcal{V}^{1 / 3}}\right) \mathcal{V}^{1 / 6+n}} \\
\partial K \partial \bar{K} K^{-1} & \sim 3\left|W_{0}\right|^{2}+W_{0}^{2} \frac{A \xi+B|\tilde{\Phi}|^{2}}{\tau_{b}^{3 / 2}}
\end{aligned}
$$


where the coefficients $A$ and $B$ are introduced to keep the overall structure feasible. $\tau_{b}$ denotes the power suppression with respect to the large Kähler moduli.

We can now determine the implicit dependence of $\rho$ on the Kähler moduli in the same way as before to be $n=5 / 18$. This enables us to integrate out the matter fields completely and we end up with the following potential with respect to the Kähler moduli

$$
V=\frac{A \tau_{3}^{1 / 2-2 m} e^{-2 a \tau_{3}}}{\mathcal{V}^{5 / 3}}-\frac{B e^{-\frac{4}{3} a \tau_{3}} \tau_{3}^{5 / 3-2 m}}{\mathcal{V}^{\frac{7}{3}+\frac{1}{9}}}+\frac{C}{\mathcal{V}^{3}}
$$

Let us compare this potential with the original 3 parameter K3 potential which was calculated in [39] to be given by

$$
V=\frac{A \sqrt{\tau_{3}} e^{-2 a \tau_{3}}}{\mathcal{V}}-\frac{B \tau_{3} e^{-a \tau_{3}}}{\mathcal{V}^{2}}-\frac{C}{\mathcal{V}^{3}}
$$

The comparison leads to exactly the same results as in the $\mathbb{P}_{[1,1,1,6,9]}$ analysis from the previous section:

- We have a marginally higher suppression with respect to the overall volume arising from the change in the leading order F-terms. In addition we have a slightly larger suppression with respect to the volume in the second term, due to the matching of $\rho$ D-term and F-term contributions with powers of the large Kähler moduli.

- In the same term we have the only other difference in the exponential suppression arising after integrating out $\rho$.

- Despite missing the analytic minimisation with respect to the volume by this slight change, we can still approximate the minimisation. We then see that we have the typical exponential hierarchy between the volume and the blow-up Kähler modulus.

- To stabilise the remaining large Kähler modulus we can still use loop corrections as discussed in [39].

Due to the same change in the potential, we assume at this stage that it is possible to stabilise the Kähler moduli in the same fashion with the additional uplifting as in the Swiss-cheese case.

\subsection{Fibre Inflation}

Following the successful embedding of D-term uplifting to the 3-Parameter K3 fibration it is natural to raise the question whether we can embed fibre inflation [19] into this uplifting scenario. In order to realise the proposal of fibre inflation we need to wrap branes around the two large cycles $\tau_{1}$ and $\tau_{2}$. These additional branes do not intersect with the blow-up cycle and do not create additional matter field content at the intersections. To obtain the potential of fibre inflation, one has to study the loop corrections $\left(g_{s}\right)$ to the scalar potential. As shown in section 3.1.2 of [19] the leading order string-loop corrections are then given by the following contributions: 
- From the branes wrapping the 4-cycle $\tau_{1}$ :

$$
\delta V_{\left(g_{s}\right), \tau_{1}}^{\mathrm{KK}}=\frac{g_{s}^{2} W_{0}^{2}\left(C_{1}^{\mathrm{KK}}\right)^{2}}{\tau_{1}^{2} \mathcal{V}^{2}}
$$

- From the branes wrapping the 4-cycle $\tau_{2}$ :

$$
\delta V_{\left(g_{s}\right), \tau_{2}}^{\mathrm{KK}}=\frac{2 g_{s}^{2} W_{0}^{2}\left(C_{2}^{\mathrm{KK}}\right)^{2}}{\tau_{2}^{2} \mathcal{V}^{2}}
$$

- From the intersections of the two stacks of branes around $\tau_{1}$ and $\tau_{2}$ :

$$
\delta V_{\left(g_{s}\right), \tau_{1} \tau_{2}}^{\mathrm{KK}}=-\frac{2 C_{12}^{W}}{\sqrt{\tau_{1}}} \frac{W_{0}^{2}}{\mathcal{V}^{3}}
$$

- From the branes wrapping the blow-up cycle:

$$
\delta V_{\left(g_{s}\right), \tau_{3}}^{\mathrm{KK}}=\frac{g_{s}^{2} W_{0}^{2}\left(C_{3}^{\mathrm{KK}}\right)^{2}}{\sqrt{\tau_{3}} \mathcal{V}^{3}}
$$

This contribution does not depend on the "flat"-direction $\tau_{1}$ and can be hence understood as a subleading correction in the $\alpha^{\prime}$-corrections.

With those results, we can establish our additional inflationary potential on top of the leading order scalar potential of the previous section:

$$
\begin{aligned}
V & =\frac{A \tau_{3}^{1 / 2-2 m} e^{-2 a \tau_{3}}}{\mathcal{V}^{5 / 3}}-\frac{B e^{-\frac{4}{3} a \tau_{3}} \tau_{3}^{5 / 3-2 m}}{\mathcal{V}^{\frac{7}{3}+\frac{1}{9}}}+\frac{C}{\mathcal{V}^{3}}+V_{\mathrm{inf}} \\
& =\frac{A \tau_{3}^{1 / 2-2 m} e^{-2 a \tau_{3}}}{\mathcal{V}^{5 / 3}}-\frac{B e^{-\frac{4}{3} a \tau_{3}} \tau_{3}^{5 / 3-2 m}}{\mathcal{V}^{\frac{7}{3}+\frac{1}{9}}}+\frac{C}{\mathcal{V}^{3}}+\frac{W_{0}^{2}}{\mathcal{V}^{2}}\left(\frac{D}{\tau_{1}^{2}}-\frac{E}{\mathcal{V} \sqrt{\tau_{1}}}+\frac{F \tau_{1}}{\mathcal{V}^{2}}\right)
\end{aligned}
$$

We see that as in the discussion of Kähler moduli inflation both parts of the potential simply decouple. Exactly as in the original discussion of fibre inflation it is possible to stabilise the volume in a first step and then look at the $\tau_{1}$ direction as the inflationary direction. We can therefore say that it is straight forward to embed fibre inflation into the uplifted scenario and under the assumption of a constant volume the analysis of inflationary parameters will give exactly the same results. Although a multi-field analysis (i.e. taking the volume to be non-constant) is out of the scope of this article, we can still comment on the stability of our uplifted scenario with regard to variations of the volume. It was observed in the analysis of the fibre inflation model that it is necessary to avoid a runaway in the volume to introduce an uplifting term proportional to $1 / \mathcal{V}^{4 / 3}$. In our scenario, we exactly have such an uplifting term before integrating out $\rho$, since, as previously discussed, the uplifting is caused by

$$
V_{\mathrm{up}} \approx \frac{|\tilde{\rho}|^{4}}{\mathcal{V}^{22 / 9}}=\frac{|\rho|^{4}}{\mathcal{V}^{4 / 3}}
$$

We therefore expect that the multi-field analysis would give exactly the same results as in the original analysis. 


\section{Constraints for metastable dS vacua in supergravity setups}

In recent years it was studied under which general constraints it is possible to obtain metastable dS vacua and/or inflation in a general supergravity framework [20,40-42]. Since our construction from previous sections is presented in a fully $\mathcal{N}=1$ supergravity framework we would like to comment on why our approach satisfies these constraints.

These studies fall into the following two categories:

1. The first type of constraints was developed by Gomez-Reino and Scrucca [40, 42]. Subject to a vanishing cosmological constant, they developed necessary but not sufficient constraints for the existence of vacua in a general supergravity setup consisting of D-term and F-term potential.

2. In more recent papers Covi et al. [20,41] studied explicitly the possibilities for various string compactifications to obtain metastable dS minima and inflation. However, the constraints used here do not assume a vanishing cosmological constant but do not include a D-term potential.

In order to avoid a long calculation and introduction of terminology to the reader we simply would like to argue why our approach falls into the category of string models discussed in $[20,41]$ and why this also allows us to satisfy the modified constraints after the inclusion of D-term potentials:

First of all, our model heavily relies on both components of the scalar potential, D-term and F-term. Since we are in principle able to tune the minimum of our potential to zero cosmological constant, we assume that we can use the constraints developed in the first series of papers by Gomez-Reino and Scrucca. The constraints we have to satisfy are:

$$
\begin{aligned}
f^{i} f_{i}+d^{a} d_{a}= & 1 \\
R_{i \bar{j} p \bar{q}} f^{i} f^{\bar{j}} f^{p} f^{\bar{q} \leq} & \frac{2}{3}+\frac{2}{3}\left(M_{a b}^{2} / m_{3 / 2}^{2}-2 h_{a b}\right) d^{a} d^{b}+2 h^{c d} h_{a c i} h_{b d \bar{j}} f^{i} f^{\bar{j}} d^{a} d^{b} \\
& -\left(2 h_{a b} h_{c d}-h_{a b}^{i} h_{c d i}\right) d^{a} d^{b} d^{c} d^{d}+\sqrt{\frac{3}{2}} \frac{Q_{a b c}}{m_{3 / 2}} d^{a} d^{b} d^{c}
\end{aligned}
$$

where $f_{i}$ and $d_{a}$ are the F-term or respectively D-term rescaled by $1 / m_{3 / 2}$ and $R_{i \bar{j} p \bar{q}}$ denotes the Riemann tensor with respect to the Kähler metric. $h_{a b}$ is the gauge kinetic function and $Q_{a b c}$ is the variation of the gauge kinetic function with respect to the generators of the gauge symmetries. ${ }^{8}$

Neglecting the D-term for the moment, the second constraint simplifies to

$$
R_{i \bar{j} p \bar{q}} f^{i} f^{\bar{j}} f^{p} f^{\bar{q}} \leq \frac{2}{3}
$$

which was renamed in the second series of papers to $\sigma=R_{i \bar{j} p \bar{q}} f^{i} f^{\bar{j}} f^{p} f^{\bar{q}}-\frac{2}{3}>0$. For various types of string compactifications, the value of $\sigma$ was determined in the work by Covi et al. In

\footnotetext{
${ }^{8} \mathrm{~A}$ more detailed explanation of the quantities in those constraints and a derivation of those can be found in the original papers.
} 
particular it was shown that for no-scale models with included $\alpha^{\prime}$-corrections, it is always possible to satisfy this constraint for a vanishing cosmological constant (cf. equation (4.33) in [41]).

Including D-terms can even alleviate this problem, as shown in [40,42] since one can rescale the F-terms and the curvature in such a way that the dependence on the D-terms seems to disappear:

$$
\begin{aligned}
\delta_{I \bar{J}} z^{I} z^{\bar{J}} & =1, \\
\tilde{R}_{I \bar{J} P \bar{Q}} z^{I} z^{\bar{J}} z^{P} z^{\bar{Q}} & \leq \frac{2}{3},
\end{aligned}
$$

where $z^{I}=f^{I} / \sqrt{1-\sum_{A} d_{A}^{2}}$. The change in the curvature can be evaluated in particular limits of the relation between gaugino and gravitino mass. In the terminology of those papers, we are working in the regime of the "light vector limit" since $g_{U(1)} M_{U(1)} / 2 m_{3 / 2}<$ $g_{U(1)} \ll 1$. In this case it was shown in [42] that the curvature is lowered due to the D-terms. Hence we can conclude that in our class of models we are easily able to satisfy the constraints for general supergravity setups and the possibility of generating stable dS minima.

\subsection{Constraints on Anomalous $U(1)$ Gauge Symmetries as Uplifting Potential}

Our explicit construction for $\mathrm{dS}$ moduli stabilisation with the help of an anomalous $U(1)$ gauge symmetries contrasts with the findings of Choi and Jeong in [43]. In this article they consider the possibility of using the D-term potential of an anomalous $U(1)$ symmetry as the uplifting mechanism and find that no uplifting is possible if the gravitino mass is smaller than the Planck scale by many orders of magnitude, assuming the Kähler moduli of order unity. This assumption is clearly violated in the context of the LARGE volume scenario discussed here which then allows to have D-term uplifting. Recall also that, unlike the KKLT scenario for which the original F-term vanishes, the LARGE volume scenario has non-vanishing F-terms and therefore non-vanishing D-terms are also possible.

\section{Conclusions}

In this article we have found a probably unexpected application of the ISS scenario. Embedding it within low-energy effective actions from type IIB string compactifications, the original metastable minimum of the ISS scenario tends to be destabilised towards runaway in the direction of the Kähler modulus determining the gauge coupling constants. This happens even after introducing a constant flux induced superpotential $W_{0}$ in the simplest one-modulus case.

Things change dramatically in Swiss-cheese compactifications with several Kähler moduli. We have found that in these cases, the effective potential for the Kähler moduli, obtained after matter field stabilisation has a similar form to the original LARGE volume scenario. Contrary to that case, in which the non-perturbative superpotential is assumed not to depend on chiral matter fields, the scalar potential cannot be minimised analytically. But the similarity allows for a numerical treatment that detects an exponentially large volume, with several differences. The main difference is that the necessary presence 
of D-terms, induced by anomalous $U(1)$ 's gives a positive definite contribution to the scalar potential in such a way that well inside the Kähler cone $\left(\tau_{1} \gg \tau_{2}\right)$ the potential goes to zero from above. Therefore this allows naturally to de Sitter compactifications with exponentially large volume. Using the fluxes, the minimum of the potential can be tuned to essentially zero value.

This allows for interesting applications: First, for the computation of soft supersymmetry breaking terms in a realistic context in which the ISS scenario plays the role of hidden sector and the Standard Model brane wraps a cycle without non-perturbatively induced superpotential. The soft terms are universal as in the standard LARGE volume scenario since the fields that break supersymmetry are the Kähler moduli which unlike the complex structure moduli, are insensitive to flavour. Generically the soft terms will be all of order the gravitino mass, but cancelations are possible depending on the modular weights of the matter fields.

Secondly, our scenario allows also the possibility of realising Kähler moduli inflation and Fibre Inflation in a fully supersymmetric set-up, the previous realisations were obtained using an ad-hoc uplifting term. Essentially we used the fact that we can obtain de Sitter space to provide the positive contribution to the scalar potential and then use a different Kähler modulus as the inflaton, just as in the original formulations of both scenarios. A full analysis of the multi-field system for inflation is out of the scope of this article.

This scenario appears to be quite general. The main fact we used is the existence of an anomalous $U(1)$ with its D-term potential and a nonperurbative contribution to the superpotential of the form $e^{-a T} \Phi \rho$. Therefore we expect the lifted large volume minimum to appear in a large class of chiral models for which the non-perturbatively induced superpotential includes matter fields. An interesting open question is to embed this scenario within a realistic compact Calabi-Yau construction.

\section{Acknowledgements}

We would like to thank Cliff Burgess, Michele Cicoli, Joe Conlon, Matt Dolan, Marta Gomez-Reino, Anshuman Maharana and Angel Uranga for useful discussions. SLK is funded by SDW, ETC and EPSRC. FQ is funded by STFC.

\section{A. On stabilising the F-term potential with a constraint}

In the process of stabilising the D-term and F-term potential separately, we claimed that it is not possible to stabilise all fields in a suitable regime (e.g. at large volume). We now would like to prove this result. The starting point is the schematic potential

$$
V=A|\rho \Phi|^{2}+2 B \operatorname{Re}(\rho \Phi)+C\left(\xi+|\rho|^{2}+|\Phi|^{2}\right),
$$

where the coefficients depend on all other variables and constants. In addition we have the constraint from the minimisation of the D-term potential.

$$
|\rho|^{2}=x|\Phi|^{2}+b,
$$


where $b$ is determined by the FI-contribution to the D-term potential and $x$ denotes the arbitrariness in the charge assignment. Differentiating with respect to $\rho$ and $\Phi$ leads to the following two equations:

$$
\begin{aligned}
& 0=A \rho^{*}|\Phi|^{2}+B \Phi+C \rho^{*} \\
& 0=A \Phi^{*}|\rho|^{2}+B \rho+C \Phi^{*}
\end{aligned}
$$

Multiplying the first equation by $\rho$ and the second by $\Phi$ and subtracting, this gives $|\Phi|=|\rho|$, which cannot be satisfied since the D-term implies explicitly that they cannot be equal in order to cancel the FI term.

\section{B. Next to leading order corrections to $\Phi$}

So far we stabilised $\rho$ and $\Phi$ to leading order. Since the stabilisation with respect to $\rho$ depends crucially on the cancellation of the FI term, one can ask what happens if we want to stabilise $\Phi$ up to next to leading order. Does this destabilise the whole stabilisation procedure? In order to answer this question, let us repeat the ansatz for $\Phi$ :

$$
\Phi=\frac{1}{\mathcal{V}^{1 / 6}}\left(\tilde{\Phi}+\frac{\varphi}{\mathcal{V}^{\beta}}\right)
$$

and the next to leading order contribution to $\partial_{\tilde{\Phi}} V$ at the leading order value:

$$
\begin{aligned}
\left.\partial_{\tilde{\Phi}} V\right|_{\tilde{\Phi}=\tilde{\Phi}+\varphi / \mathcal{V}^{\beta}} & =\frac{2}{2 \tau_{2} \mathcal{V}^{2}}\left(\frac{3 \sqrt{\tau_{2}} \xi}{2 a \mathcal{V}}+\frac{2 \tilde{\Phi} \frac{\varphi}{\mathcal{V}^{\beta}}\left(18 a \tau_{2}-n\right)}{18 a \tau_{2}^{1-n}}\right) \tilde{\Phi} \\
& -\frac{2}{2 \tau_{2} \mathcal{V}^{5 / 2}}\left(\frac{2 \tilde{\Phi}\left(18 a \tau_{2}-n\right)}{18 a \tau_{2}^{1-n}}\right)\left(\frac{|\tilde{\rho}|^{2}\left(36 a \tau_{2}+n\right)}{18 a \tau_{2}^{1-n}}\right) \\
& +\frac{2 \tilde{\Phi} \alpha^{2} e^{-2 a \tau_{2}}}{\tau_{2}^{n} \mathcal{V}^{5 / 3}} .
\end{aligned}
$$

In our scenario $\varphi$ is taking negative or small values which can be seen as follows: At the LARGE volume minimum, we expect

$$
e^{a \tau_{2}} \sim \mathcal{V}
$$

This implies that the contribution proportional to $\tilde{\rho}$ is suppressed with an additional factor $1 / \mathcal{V}^{2 / 3}$ which makes it subleading compared to the next to leading order corrections coming from the FI bit. Hence, $\beta=1$ and the next to leading order corrections to $\Phi$ at our minimum

will be negative. Suppose $e^{a \tau_{2}}$ is not suppressed with respect to the volume, the contribution from the leading order F-term will be dominating and drives $\varphi$ to negative values again. In an intermediate range the contribution from the cross-term between $\rho$ and $\Phi$ might be leading. However, we were never able to assign $\varphi$ a value to cancel our D-term contribution completely and hence we always keep the uplifting contribution. 


\section{Minimizing quark masses}

\section{1-modulus}

In principle there is a flat direction in the leading order D-term potential which allows nonvanishing $q$ and $p$. So we can minimize the D-term potential for non-vanishing $p, q$ and then we have to try to minimize the F-term potential. Looking at the only non-trivial case of no implicit suppression with respect to the large Kähler modulus, the leading order F-term contribution is given by

$$
A\left|\frac{p \Phi q}{\mu}+\rho \Phi\right|^{2}
$$

where A contains constants and the dependence on the Kähler modulus. The first derivative with respect to $p$ gives

$$
A \Phi q\left(\frac{p \Phi q}{\mu}+\rho \Phi\right)^{*}
$$

which is only zero for non-vanishing $\Phi$ and $\rho$ if $q=0$, since $p q / \mu+\rho \neq 0$ due to the rank condition. The case of an implicit suppression with respect to the Kähler moduli is discussed below.

\section{2-moduli}

A priori it is clear that the potential is extremized at $p=q=0$ since the dominating D-term potential is extremized. Suppose this extremum corresponds to a maximum and we could find a minimum closeby. At that minimum $p$ has an implicit dependence on the large Kähler modulus. This dependence has to be at least of the suppression of $\Phi$ (i.e. $1 / \tau_{1}^{1 / 4}$ ). Schematically the D-term potential looks like

$$
V_{D} \sim\left(|\Phi|^{2}-|\rho|^{2}-|p|^{2}-\mathrm{FI}\right) .
$$

Neglecting $\rho$ for the moment, we can see that there is a flat direction in the D-term potential corresponding to $|\Phi|^{2}-\mathrm{FI}=|p|^{2}$. Looking at next to leading order effects, i.e. the F-term potential in this case we can "lift" this flat direction. Since we are perturbing around our solution presented in the main part $|\Phi|^{2}-\mathrm{FI} \approx 0$ and in particular it should be suppressed with respect to the volume at least as much as $|\Phi|$. Then the leading order contribution from the F-term potential still is given by

$$
\frac{|\Phi|^{2} e^{-2 a \tau_{2}}}{\tau_{1}^{5 / 2}} .
$$

Hence we do not want $|\Phi|$ to become larger at next to leading order, which implies that $|p|=0$ is a minimum.

\section{Estimating scales}

The masses associated with our fields are given as the square root of the eigenvalues of the matrix

$$
K_{i \bar{j}}^{-1} \frac{\partial^{2} V}{\partial x^{i} \partial x^{\bar{j}}}
$$


To understand the results it is very helpful to estimate the leading order contributions to the Kähler metric which we find to be

$$
K_{i \bar{j}}\left(\tau_{1}, \tau_{2}, \rho, \Phi\right)=\left(\begin{array}{cccc}
\frac{3 M_{P}^{2}}{4 \tau_{1}^{2}} & -\frac{27 M_{P}^{2} \tau_{2}^{7 / 6}+2 g_{s}^{-2 / 3}|\tilde{\Phi}|^{2}}{24 \tau_{1}^{5 / 2} \tau_{2}^{2 / 3}} & -\frac{g_{s}^{-2 / 3} \tilde{\rho} \tau_{2}^{1 / 3}}{2 \tau_{1}^{2 / 12}} & -\frac{g_{s}^{-2 / 3} \tau_{2}^{1 / 3} \tilde{\Phi}}{2 \tau_{1}^{9 / 4}} \\
-\frac{27 M_{P}^{2} \tau_{2}^{7 / 6}+2 g_{s}^{-2 / 3}|\tilde{\Phi}|^{2}}{24 \tau_{1}^{5 / 2} \tau_{2}^{2 / 3}} & \frac{27 M_{P}^{2} \tau_{2}^{7 / 6}-4 g_{s}^{-2 / 3}|\tilde{\Phi}|^{2}}{72 \tau_{1}^{3 / 2} \tau_{2}^{5 / 3}} & \frac{g_{s}^{-2 / 3} \tilde{\rho}}{6 \tau_{1}^{17 / 12} \tau_{2}^{2 / 3}} & \frac{g_{s}^{-2 / 3} \tilde{\Phi}}{6 \tau_{1}^{5 / 4} \tau_{2}^{2 / 3}} \\
-\frac{g_{s}^{-2 / 3} \tilde{\rho}^{*} \tau_{2}^{1 / 3}}{2 \tau_{1}^{29 / 12}} & \frac{g_{s}^{-2 / 3} \tilde{\rho}^{*}}{6 \tau_{1}^{17 / 12} \tau_{2}^{2 / 3}} & \frac{g_{s}^{-2 / 3} \tau_{2}^{1 / 3}}{\tau_{1}} & 0 \\
-\frac{\tau_{2}^{1 / 3} \tilde{\Phi}^{*}}{2 g_{s}^{2 / 3} \tau_{1}^{9 / 4}} & \frac{\tilde{\Phi}_{2}^{*}}{6 g_{s}^{2 / 3} \tau_{1}^{5 / 4} \tau_{2}^{2 / 3}} & 0 & \frac{g_{s}^{-2 / 3} \tau_{2}^{1 / 3}}{\tau_{1}}
\end{array}\right)
$$

This leading order behaviour in the Kähler metric is with respect to the the Kähler moduli almost the same as in the LARGE volume case. The only change is given by the contribution proportional to $\Phi$ in the relevant components but this change is negligibly small.

We find the leading order volume suppression in the Hessian of the potential evaluated at the minimum for the brane moduli to be:

$$
d^{2} V=\left(\begin{array}{cccc}
\frac{1}{\tau_{1}^{5}} & \frac{1}{\tau_{1}^{3+1 / 3}} & \frac{1}{\tau_{1}^{4+5 / 12}} & \frac{1}{\tau_{1}^{3+3 / 4}} \\
\frac{1}{\tau_{1}^{3+1 / 3}} & \frac{1}{\tau_{1}^{3}} & \frac{1}{\tau_{1}^{3+5 / 12}} & \frac{1}{\tau_{1}^{2+3 / 4}} \\
\frac{1}{\tau_{1}^{4+5 / 12}} & \frac{1}{\tau_{1}^{3+5 / 12}} & \frac{1}{\tau_{1}^{3+5 / 6}} & \frac{1}{\tau_{1}^{2+2 / 3}} \\
\frac{1}{\tau_{1}^{3+3 / 4}} & \frac{1}{\tau_{1}^{3+3 / 4}} & \frac{1}{\tau_{1}^{2+2 / 3}} & \frac{1}{\tau_{1}^{2+1 / 2}}
\end{array}\right)
$$

where we assumed that at $e^{a \tau_{2}} \sim \tau_{1}^{3 / 2}$ and we neglected the exact coefficients for simplicity. Numerically this leads to the following masses for the dS example from the previous section:

$$
\begin{aligned}
m_{\tau_{1}}^{2} & =6.7 \times 10^{-7} M_{P}^{2} \\
m_{\tau_{2}}^{2} & =2.5 \times 10^{-6} M_{P}^{2} \\
m_{\rho}^{2} & =2.7 \times 10^{-7} M_{P}^{2} \\
m_{\Phi}^{2} & =3.9 \times 10^{-5} M_{P}^{2}
\end{aligned}
$$

In order to interpret these results we would like to compare the masses with the masses in the LARGE volume scenario, which are given by

$$
\begin{aligned}
& m_{\tau_{1}} \sim \frac{g_{s}^{2} W_{0}}{\left(\mathcal{V}_{s}^{0}\right)^{3 / 2}} M_{P} \sim m_{3 / 2}\left(\frac{m_{3 / 2}}{M_{P}}\right)^{1 / 2}, \\
& m_{\tau_{2}} \sim \frac{a g_{s} W_{0}}{\mathcal{V}_{s}^{0}} M_{P} \sim 2 m_{3 / 2} \ln M_{P} / m_{3 / 2} .
\end{aligned}
$$

Taking now the same parameters as for the dS example, we obtain

$$
\begin{aligned}
& m_{\tau_{1}}^{2}=4.1 \times 10^{-8} M_{P}^{2}, \\
& m_{\tau_{2}}^{2}=1.2 \times 10^{-5} M_{P}^{2} .
\end{aligned}
$$

Compared with the original LARGE volume scenario, we can conclude that we obtain roughly the same masses for the Kähler moduli. One of the brane moduli becomes very 
massive $m_{\Phi}$ and the other mass $m_{\rho}$ is relatively the lightest particle but it is roughly of the same order as the large Kähler modulus $\tau_{1}$. The difference in the Kähler moduli masses is due to different numerical prefactors in the volume of the Calabi-Yau. In order to compare these results completely reliably, we would have to go to a realistic value of the volume which goes beyond our simple dS example. Before such an analysis is possible we should estimate further crucial properties of this modified model such as the structure of supersymmetry breaking.

\section{E. An approximative analytic stabilisation of the large Kähler modulus}

After integrating out the matter field degrees of freedom we ended up with the following potential

$$
V=g_{s} M_{P}^{4}\left(\frac{A e^{-2 a \tau_{2}}}{\tau_{1}^{5 / 2} \tau_{2}^{1 / 6}}-\frac{B e^{-4 / 3 a \tau_{2}} \tau_{2}^{14 / 9}}{\tau_{1}^{11 / 3}}+\frac{C}{\tau_{1}^{9 / 2}}\right) .
$$

Changing the suppression with respect to the large Kähler modulus $\tau_{1}$ in the term proportional to $B$ from $11 / 3$ by $1 / 6$ to $7 / 2$ should not affect the structure qualitatively. The change in the minimal value is negligibly small but allows us to minimise the potential analytically.

The modified potential takes the classical form of the volume suppression looking like:

$$
V=\frac{g_{s} M_{P}^{4}}{\tau_{1}^{9 / 2}}\left(\frac{A e^{-2 a \tau_{2}} \tau_{1}^{2}}{\tau_{2}^{1 / 6}}-B e^{-4 / 3 a \tau_{2}} \tau_{2}^{14 / 9} \tau_{1}+C\right)
$$

Demanding the vanishing of the first derivative of the potential with respect to $\tau_{1}$ leads to

$$
\begin{aligned}
0=\frac{\partial V}{\partial \tau_{1}} & =-\frac{g_{s} M_{P}^{4}}{2 \tau_{1}^{11 / 2}}\left(\frac{5 A e^{-2 a \tau_{2}} \tau_{1}^{2}}{\tau_{2}^{1 / 6}}-7 B e^{-4 / 3 a \tau_{2}} \tau_{2}^{14 / 9} \tau_{1}+9 C\right) \\
\Rightarrow 0 & =\tau_{1}^{2}-\frac{7 B e^{2 / 3 a \tau_{2}} \tau_{2}^{31 / 18}}{5 A} \tau_{1}+\frac{9 C e^{2 a \tau_{2}} \tau_{2}^{1 / 6}}{5 A} \\
& =\tau_{1}^{2}-B^{\prime} e^{2 / 3 a \tau_{2}} \tau_{1}+C^{\prime} e^{2 / 3 a \tau_{2}} .
\end{aligned}
$$

Solutions to this equation are given by

$$
\tau_{1}^{(\min , \max )}=\frac{B^{\prime}}{2} e^{2 / 3 a \tau_{2}} \pm \sqrt{\frac{B^{\prime 2}}{4} e^{4 / 3 a \tau_{2}}-C^{\prime} e^{2 a \tau_{2}}} .
$$

Looking at the leading order behaviour of the overall potential, it is clear that the negative solution will correspond to the minimum and the positive solution to the maximum. In order to keep to real solutions we have to satisfy the following condition for the discriminant:

$$
\frac{B^{\prime 2}}{4 C^{\prime}}>e^{2 / 3 a \tau_{2}}
$$

The value of the discriminant is also crucial for the value we can stabilise the small Kähler modulus $\tau_{2}$ at: 
Demanding the vanishing of the first derivative with respect to the small Kähler modulus leads to the following condition:

$$
0=4 B e^{2 / 3 a \tau_{2}} \tau_{2}^{31 / 18}\left(-7+6 a \tau_{2}\right)-3 A \tau_{1}\left(1+12 a \tau_{2}\right),
$$

which is equivalent to writing

$$
0=\frac{20}{21} B^{\prime} e^{2 / 3 a \tau_{2}}\left(-7+6 a \tau_{2}\right)-\tau_{1}\left(1+12 a \tau_{2}\right)
$$

In the large $a \tau_{2}$ limit this condition simplifies at the minimum to the following equation

$$
\begin{aligned}
0 & =-\frac{1}{21} B^{\prime} e^{2 / 3 a \tau_{2}}+2 \sqrt{\frac{B^{\prime 2}}{4} e^{4 / 3 a \tau_{2}}-C^{\prime} e^{2 a \tau_{2}}} \\
\Rightarrow \frac{440}{441} \frac{B^{\prime 2}}{4 C^{\prime}} & =e^{2 / 3 a \tau_{2}} .
\end{aligned}
$$

This is consistent with the constraint from above as expected and we obtain the same behaviour in an exact calculation.

Unfortunately, we cannot solve the remaining equations for $\tau_{2}$ analytically. However, we can be sure that a solution exists if the constraint is satisfied. Furthermore these approximations should help in constructing solutions numerically.

\section{F. Calculating F-terms}

Using $D_{S} W=0$, the F-term associated with the dilaton field $S$ is found to be given by at leading order:

$$
\begin{aligned}
F^{\bar{s}} & =e^{\frac{K}{2 M_{P}^{2}}}\left(K^{\bar{s} \tau_{i}} D_{\tau_{i}} W+K^{\bar{s} \phi_{i}} D_{\phi_{i}} W\right) \\
& =\frac{1}{g_{s} \mathcal{V}}\left(K^{\bar{s} \tau_{i}} \partial_{\tau_{i}} W+\frac{W}{M_{P}^{2}} K^{\bar{s} \tau_{i}} \partial_{\tau_{i}} K+K^{\bar{s} \phi_{i}} \partial_{\phi_{i}} W+\frac{W}{M_{P}^{2}} K^{\bar{s} \phi_{i}} \partial_{\phi_{i}} K\right) \\
& =\frac{1}{g_{s} \mathcal{V}}\left(K^{\bar{s} \tau_{2}} \partial_{\tau_{2}} W+M_{P} g_{s}^{3 / 2} W_{0} K^{\bar{s} \tau_{1}} \partial_{\tau_{1}} K+K^{\bar{s} \Phi} \partial_{\Phi} W+K^{\bar{s} \rho} \partial_{\rho} W\right) \\
& =\frac{1}{g_{s} \mathcal{V}}\left(\frac{18 \sqrt{2} M_{P} g_{s}^{3 / 2} W_{0} s^{5 / 2} \xi}{\tau_{1}^{3 / 2}}+\frac{36 \sqrt{2} e^{-a \tau_{2}} g^{1 / 2+m} s^{5 / 2} \alpha a \xi \tilde{\rho} \tilde{\Phi}}{\tau_{1}^{13 / 6}}\right) \\
& =\frac{18 \sqrt{2} M_{P} g_{s}^{1 / 2} W_{0} s^{5 / 2} \xi}{\mathcal{V}^{2}}+O\left(\frac{1}{\mathcal{V}^{22 / 9}}\right),
\end{aligned}
$$

which is the same as in the LVS. The F-terms associated with the Kähler moduli are at 
leading order given by:

$$
\begin{aligned}
& F^{\bar{\tau}_{1}}=e^{\frac{K}{2 M_{P}^{2}}}\left(K^{\bar{\tau}_{1} \tau_{i}} D_{\tau_{i}} W+K^{\bar{\tau}_{1} \phi_{i}} D_{\phi_{i}} W\right) \\
& =\frac{1}{g_{s}\left(\mathcal{V}+\frac{\xi}{g_{s}^{3 / 2}}\right)}\left(K^{\bar{\tau}_{1} \tau_{i}} \partial_{\tau_{i}} W+\frac{W}{M_{P}^{2}} K^{\bar{\tau}_{1} \tau_{i}} \partial_{\tau_{i}} K+K^{\bar{\tau}_{1} \phi_{i}} \partial_{\phi_{i}} W+\frac{W}{M_{P}^{2}} K^{\bar{\tau}_{1} \phi_{i}} \partial_{\phi_{i}} K\right) \\
& =\frac{1}{g_{s}\left(\mathcal{V}+\frac{\xi}{g_{s}^{3 / 2}}\right)}\left(-2 W_{0} M_{P} g_{s}^{3 / 2} \tau_{1}+K^{\bar{\tau}_{1} \tau_{i}} \partial_{\tau_{i}} W+K^{\bar{\tau}_{1} \phi_{i}} \partial_{\phi_{i}} W\right) \\
& =\frac{1}{g_{s}\left(\mathcal{V}+\frac{\xi}{g_{s}^{3 / 2}}\right)}\left(-2 W_{0} M_{P} g_{s}^{3 / 2} \tau_{1}-\frac{6 \sqrt{2} W_{0} M_{P} \xi}{\sqrt{\tau_{1}}}+\frac{4 a \alpha g_{s}^{1 / 2+m}}{M_{P}} \tilde{\rho} \tilde{\Phi} \tau_{1}^{1 / 3} \tau_{2} e^{-a \tau_{2}}(\bar{F}, 2)\right.
\end{aligned}
$$

where unlike in the LVS the third term is dominating over the second one at the minimum. In the LVS as discussed in [34] there is a sub-leading cancellation, which is not present due to a different leading order structure of the potential and the inclusion of the D-term uplifting contribution: Here the second term plus the D-term uplifting contribution (with opposite sign) are of the order of the second one, cf. equation (4.18):

$$
V \supset \frac{2 \tau_{2}^{2 n-1}|\tilde{\rho}|^{4}}{g_{s}^{1 / 3} \mathcal{V}^{22 / 9}}+\frac{2 \sqrt{6} M_{P}^{3} g_{s}^{2 / 3} W_{0} \alpha \sqrt{6} \tau_{2}^{5 / 4-n / 2} e^{-a \tau_{2}} \operatorname{Re} \tilde{\rho}}{\mathcal{V}^{22 / 9}}+\frac{3 M_{P}^{4} W_{0}^{2}}{2 \mathcal{V}^{27 / 9}}\left(\frac{\xi}{g_{s}^{3 / 2}}+\frac{n \sqrt{\tau_{2}}}{a}\right)
$$

With an absent D-term uplifting contribution the third term becomes dominant. However, it is out of the scope of this article to determine the precise volume suppression of the F-term when the second and third term cancel themselves partially.

$$
\begin{aligned}
F^{\overline{\tau_{2}}} & =e^{\frac{K}{2 M_{P}^{2}}}\left(K^{\bar{\tau}_{2} \tau_{i}} D_{\tau_{i}} W+K^{\bar{\tau}_{2} \phi_{i}} D_{\phi_{i}} W\right) \\
& =\frac{1}{g_{s}\left(\mathcal{V}+\frac{\xi}{g_{s}^{3 / 2}}\right)}\left(K^{\bar{\tau}_{2} \tau_{i}} \partial_{\tau_{i}} W+\frac{W}{M_{P}^{2}} K^{\bar{\tau}_{2} \tau_{i}} \partial_{\tau_{i}} K+K^{\bar{\tau}_{2} \phi_{i}} \partial_{\phi_{i}} W+\frac{W}{M_{P}^{2}} K^{\bar{\tau}_{2} \phi_{i}} \partial_{\phi_{i}} K\right) \\
& =\frac{1}{g_{s}\left(\mathcal{V}+\frac{\xi}{g_{s}^{3 / 2}}\right)}\left(-2 M_{P} W_{0} g_{s}^{3 / 2} \tau_{2}-\frac{8 a \alpha g_{s}^{1 / 2+n} \tilde{\rho} \tilde{\Phi} \tau_{1}^{5 / 6} \sqrt{\tau_{2}} e^{-a \tau_{2}}}{3 M_{P}}\right), \\
F^{\overline{\tau_{3}}} & =e^{\frac{K}{2 M_{P}^{2}}\left(K^{\bar{\tau}_{3} \tau_{i}} D_{\tau_{i}} W+K^{\bar{\tau}_{3} \phi_{i}} D_{\phi_{i}} W\right)} \\
= & \frac{1}{g_{s}\left(\mathcal{V}+\frac{\xi}{g_{s}^{3 / 2}}\right)}\left(K^{\bar{\tau}_{3} \tau_{i}} \partial_{\tau_{i}} W+\frac{W}{M_{P}^{2}} K^{\bar{\tau}_{3} \tau_{i}} \partial_{\tau_{i}} K+K^{\bar{\tau}_{3} \phi_{i}} \partial_{\phi_{i}} W+\frac{W}{M_{P}^{2}} K^{\bar{\tau}_{3} \phi_{i}} \partial_{\phi_{i}} K\right) \\
= & \frac{1}{g_{s}\left(\mathcal{V}+\frac{\xi}{g_{s}^{3 / 2}}\right)}\left(-2 M_{P} W_{0} g_{s}^{3 / 2} \tau_{3}-\frac{4 a \alpha g_{s}^{1 / 2+m} \tilde{\rho} \tilde{\Phi} \tau_{2} \tau_{3} e^{-a \tau_{2}}}{M_{P} \tau_{1}^{2 / 3}}\right)
\end{aligned}
$$


Although it is not of importance in the analysis of the soft-term structure, we would like to mention the fact that there is no cancellation in the F-term associated with $\tau_{2}$ as in the LVS. This is due to the fact that the leading order contributions in the F-term potential come from various F-terms and not only from the small modulus $\tau_{2}$.

\section{References}

[1] S. Kachru, R. Kallosh, A. Linde, and S. P. Trivedi, De Sitter vacua in string theory, Phys. Rev. D68 (2003) 046005, hep-th/0301240.

[2] C. P. Burgess, R. Kallosh, and F. Quevedo, de Sitter string vacua from supersymmetric D-terms, JHEP 10 (2003) 056, hep-th/0309187.

[3] A. Saltman and E. Silverstein, The scaling of the no-scale potential and de Sitter model building, JHEP 11 (2004) 066, hep-th/0402135.

O. Lebedev, H. P. Nilles, and M. Ratz, de Sitter vacua from matter superpotentials, Phys.

Lett. B636 (2006) 126-131, hep-th/0603047.

L. Covi, M. Gomez-Reino, C. Gross, G. A. Palma, and C. A. Scrucca, Constructing de Sitter vacua in no-scale string models without uplifting, arXiv:0812.3864.

R. Brustein and S. P. de Alwis, Moduli potentials in string compactifications with fluxes: Mapping the discretuum, Phys. Rev. D69 (2004) 126006, hep-th/0402088.

[4] P. Binetruy, G. Dvali, R. Kallosh, and A. Van Proeyen, Fayet-Iliopoulos terms in supergravity and cosmology, Class. Quant. Grav. 21 (2004) 3137-3170, hep-th/0402046.

K. Choi, A. Falkowski, H. P. Nilles, and M. Olechowski, Soft supersymmetry breaking in KKLT flux compactification, Nucl. Phys. B718 (2005) 113-133, hep-th/0503216|.

G. Villadoro and F. Zwirner, D terms from D-branes, gauge invariance and moduli stabilization in flux compactifications, JHEP 03 (2006) 087, hep-th/0602120].

G. Villadoro and F. Zwirner, de Sitter vacua via consistent D-terms, Phys. Rev. Lett. 95 (2005) 231602, hep-th/0508167.

S. P. de Alwis, Effective potentials for light moduli, Phys. Lett. B626 (2005) 223-229, hep-th/0506266.

[5] A. Achucarro, B. de Carlos, J. A. Casas, and L. Doplicher, de Sitter vacua from uplifting D-terms in effective supergravities from realistic strings, JHEP 06 (2006) 014, hep-th/0601190.

[6] D. Cremades, M. P. Garcia del Moral, F. Quevedo, and K. Suruliz, Moduli stabilisation and de Sitter string vacua from magnetised D7 branes, JHEP 05 (2007) 100, hep-th/0701154.

[7] M. Haack, D. Krefl, D. Lust, A. Van Proeyen, and M. Zagermann, Gaugino condensates and D-terms from D7-branes, JHEP 01 (2007) 078, hep-th/0609211.

[8] K. Intriligator, N. Seiberg, and D. Shih, Dynamical SUSY breaking in meta-stable vacua, JHEP 04 (2006) 021, hep-th/0602239.

[9] A. Giveon and D. Kutasov, Gauge symmetry and supersymmetry breaking from intersecting branes, Nucl. Phys. B778 (2007) 129-158, hep-th/0703135.

A. Giveon and D. Kutasov, Stable and Metastable Vacua in Brane Constructions of SQCD, JHEP 02 (2008) 038, arXiv:0710.1833.

S. Franco, I. Garcia-Etxebarria, and A. M. Uranga, Non-supersymmetric meta-stable vacua from brane configurations, JHEP 01 (2007) 085, hep-th/0607218. 
H. Ooguri and Y. Ookouchi, Meta-stable supersymmetry breaking vacua on intersecting branes, Phys. Lett. B641 (2006) 323-328, hep-th/0607183.

R. Argurio, M. Bertolini, S. Franco, and S. Kachru, Metastable vacua and D-branes at the conifold, JHEP 06 (2007) 017, hep-th/0703236.

R. Argurio, M. Bertolini, S. Franco, and S. Kachru, Gauge/gravity duality and meta-stable dynamical supersymmetry breaking, JHEP 01 (2007) 083, hep-th/0610212.

S. Franco and A. M. . Uranga, Dynamical SUSY breaking at meta-stable minima from Dbranes at obstructed geometries, JHEP 06 (2006) 031, hep-th/0604136.

[10] C. P. Burgess et. al., The Inflationary Brane-Antibrane Universe, JHEP 07 (2001) 047, hep-th/0105204.

G. R. Dvali, Q. Shafi, and S. Solganik, D-brane inflation, hep-th/0105203.

S. Kachru et. al., Towards inflation in string theory, JCAP 0310 (2003) 013, hep-th/0308055.

M. Alishahiha, E. Silverstein, and D. Tong, DBI in the sky, Phys. Rev. D70 (2004) 123505, hep-th/0404084.

[11] K. Dasgupta, C. Herdeiro, S. Hirano, and R. Kallosh, D3/D7 inflationary model and M-theory, Phys. Rev. D65 (2002) 126002, hep-th/0203019.

C. Herdeiro, S. Hirano, and R. Kallosh, String theory and hybrid inflation / acceleration, JHEP 12 (2001) 027, hep-th/0110271.

M. Haack et. al., Update of D3/D7-Brane Inflation on $K 3 x T^{2} / Z_{2}$, Nucl. Phys. B806 (2009) 103-177, arXiv:0804.3961.

C. P. Burgess, J. M. Cline, and M. Postma, Axionic D3-D7 Inflation, arXiv:0811.1503.

[12] E. Silverstein and A. Westphal, Monodromy in the CMB: Gravity Waves and String Inflation, Phys. Rev. D78 (2008) 106003, arXiv:0803.3085.

[13] A. Avgoustidis, D. Cremades, and F. Quevedo, Wilson line inflation, Gen. Rel. Grav. 39 (2007) 1203-1234, hep-th/0606031.

A. Avgoustidis and I. Zavala, Warped Wilson Line DBI Inflation, arXiv:0810.5001.

[14] J. J. Blanco-Pillado et. al., Racetrack inflation, JHEP 11 (2004) 063, hep-th/0406230.

[15] J. J. Blanco-Pillado et. al., Inflating in a better racetrack, JHEP 09 (2006) 002, hep-th/0603129.

[16] J. P. Conlon and F. Quevedo, Kaehler moduli inflation, JHEP 01 (2006) 146, hep-th/0509012.

[17] J. R. Bond, L. Kofman, S. Prokushkin, and P. M. Vaudrevange, Roulette inflation with Kaehler moduli and their axions, Phys. Rev. D75 (2007) 123511, hep-th/0612197.

[18] L. McAllister, E. Silverstein, and A. Westphal, Gravity Waves and Linear Inflation from Axion Monodromy, arXiv:0808.0706.

[19] M. Cicoli, C. P. Burgess, and F. Quevedo, Fibre Inflation: Observable Gravity Waves from IIB String Compactifications, arXiv:0808.0691.

[20] L. Covi et. al., Constraints on modular inflation in supergravity and string theory, JHEP 08 (2008) 055, arXiv:0805.3290].

[21] V. Balasubramanian, P. Berglund, J. P. Conlon, and F. Quevedo, Systematics of moduli stabilisation in Calabi-Yau flux compactifications, JHEP 03 (2005) 007, hep-th/0502058. 
[22] J. P. Conlon, F. Quevedo, and K. Suruliz, Large-volume flux compactifications: Moduli spectrum and D3/D7 soft supersymmetry breaking, JHEP 08 (2005) 007, hep-th/0505076.

[23] K. Intriligator, N. Seiberg, and D. Shih, Supersymmetry Breaking, R-Symmetry Breaking and Metastable Vacua, JHEP 07 (2007) 017, hep-th/0703281].

[24] J. P. Conlon, D. Cremades, and F. Quevedo, Kaehler potentials of chiral matter fields for Calabi-Yau string compactifications, JHEP 01 (2007) 022, hep-th/0609180.

[25] Y. Nakayama, M. Yamazaki, and T. T. Yanagida, Moduli Stabilization in Stringy ISS Models, Phys. Lett. B663 (2008) 281-285, arXiv:0710.0001.

[26] V. Balasubramanian and P. Berglund, Stringy corrections to Kaehler potentials, SUSY breaking, and the cosmological constant problem, JHEP 11 (2004) 085, hep-th/0408054.

[27] A. Westphal, de Sitter String Vacua from Kahler Uplifting, JHEP 03 (2007) 102, hep-th/0611332.

[28] S. S. AbdusSalam, J. P. Conlon, F. Quevedo, and K. Suruliz, Scanning the Landscape of Flux Compactifications: Vacuum Structure and Soft Supersymmetry Breaking, JHEP 12 (2007) 036, arXiv:0709.0221.

[29] J. P. Conlon, S. S. Abdussalam, F. Quevedo, and K. Suruliz, Soft SUSY breaking terms for chiral matter in IIB string compactifications, JHEP 01 (2007) 032, hep-th/0610129.

[30] R. Blumenhagen, V. Braun, T. W. Grimm and T. Weigand, GUTs in Type IIB Orientifold Compactifications, Nucl. Phys. B 815 (2009) 1 [arXiv:0811.2936 [hep-th]].

[31] G. Aldazabal, L. E. Ibanez, F. Quevedo and A. M. Uranga, D-branes at singularities: A bottom-up approach to the string embedding of the standard model, JHEP 0008, 002 (2000) [arXiv:hep-th/0005067].

[32] J. P. Conlon, A. Maharana and F. Quevedo, Towards Realistic String Vacua, JHEP 0905, 109 (2009) [arXiv:0810.5660 [hep-th]].

[33] R. Blumenhagen, S. Moster, and E. Plauschinn, Moduli Stabilisation versus Chirality for MSSM like Type IIB Orientifolds, JHEP 01 (2008) 058, arXiv:0711.3389.

[34] R. Blumenhagen, J. P. Conlon, S. Krippendorf, S. Moster and F. Quevedo, SUSY Breaking in Local String/F-Theory Models, arXiv:0906.3297 [hep-th].

[35] J. P. Conlon, Moduli stabilisation and applications in IIB string theory, Fortsch. Phys. 55 (2007) 287-422, hep-th/0611039.

[36] J. P. Conlon and F. Quevedo, Gaugino and scalar masses in the landscape, JHEP 06 (2006) 029, hep-th/0605141.

[37] E. Dudas and S. K. Vempati, General soft terms from supergravity including D-terms, arXiv:hep-ph/0506029.

[38] J. P. Conlon, Mirror Mediation, JHEP 03 (2008) 025, arXiv:0710.0873.

[39] M. Cicoli, J. P. Conlon, and F. Quevedo, General Analysis of LARGE Volume Scenarios with String Loop Moduli Stabilisation, arXiv:0805.1029.

[40] M. Gomez-Reino and C. A. Scrucca, Constraints from F and D supersymmetry breaking in general supergravity theories, Fortsch. Phys. 56 (2008) 833-841, arXiv:0804.3730. 
[41] L. Covi et. al., de Sitter vacua in no-scale supergravities and Calabi-Yau string models, JHEP 06 (2008) 057, arXiv:0804.1073.

[42] M. Gomez-Reino and C. A. Scrucca, Metastable supergravity vacua with $F$ and $D$ supersymmetry breaking, JHEP 08 (2007) 091, arXiv:0706.2785.

[43] K. Choi and K. S. Jeong, Supersymmetry breaking and moduli stabilization with anomalous U(1) gauge symmetry, JHEP 06 (2006) 007, hep-th/0605108. 